\title{
Continuous flavor symmetries and the stability of asymmetric dark matter
}

\author{
Fady Bishara ${ }^{a, b}$ and Jure Zupan ${ }^{b}$ \\ ${ }^{a}$ Department of Physics, University of Cincinnati, \\ Cincinnati, Ohio 45221, U.S.A. \\ ${ }^{b}$ Theoretical Physics Department, Fermilab, \\ P.O. Box 500, Batavia, IL 60510, U.S.A.
}

E-mail: bisharfy@mail.uc.edu, zupanje@ucmail.uc.edu

ABSTRACT: Generically, the asymmetric interactions in asymmetric dark matter (ADM) models could lead to decaying DM. We show that, for ADM that carries nonzero baryon number, the continuous flavor symmetries that generate the flavor structure in the quark sector also imply a looser lower bound on the mass scale of the asymmetric mediators between the dark and visible sectors. The mediators for $B=2 \mathrm{ADM}$ that can produce a signal in the future indirect dark matter searches can thus also be searched for at the LHC. For two examples of the mediator models, with either the MFV or Froggatt-Nielsen flavor breaking pattern, we derive the FCNC constraints and discuss the search strategies at the LHC.

KEywords: Beyond Standard Model, Cosmology of Theories beyond the SM

ARXIV EPRINT: 1408.3852 


\section{Contents}

1 Introduction 1

2 Dark matter mass in asymmetric dark matter models 3

3 Metastability and flavor breaking $\quad 5$

3.1 Minimal Flavor Violation $\quad 6$

$\begin{array}{lll}3.2 & \text { Spontaneously broken horizontal symmetries } & 8\end{array}$

4 Indirect detection $\quad 10$

5 Mediator models $\quad 11$

5.1 MFV model with scalar mediators $\quad 11$

$\begin{array}{lll}5.2 & \text { FN model with fermionic and scalar mediators } & 12\end{array}$

6 Experimental signatures of the mediators $\quad 14$

$\begin{array}{lll}6.1 & \text { Flavor constraints } & 14\end{array}$

$\begin{array}{ll}\text { 6.2 Relic abundance and direct detection } & 17\end{array}$

$\begin{array}{ll}6.3 \text { Collider signatures } & 17\end{array}$

$\begin{array}{lll}7 \text { Conclusions } & 19\end{array}$

$\begin{array}{ll}\text { A Operators in four component notation } & 21\end{array}$

B Asymmetric DM relic density $\quad 22$

$\begin{array}{ll}\text { C Calculation of the DM decay time } & 24\end{array}$

$\begin{array}{ll}\text { D Loop functions in neutral meson mixing } & 28\end{array}$

\section{Introduction}

Dark matter (DM) is stable on cosmological time-scales. A principal question about the nature of DM is: what mechanism ensures its stability? Commonly, this is assumed to be a result of an exact symmetry (for a concise review of proposed stabilization mechanisms see, e.g., [1]). One possibility is that the stability of DM is ensured by a gauge symmetry mimicking the way QED gauge invariance ensures the stability of the electron in the standard model [2-4]. A more frequent choice is to introduce a $Z_{2}$ symmetry by hand. A prominent example is $R$-parity in the MSSM which both stabilizes DM and ensures the stability of the proton [5-7]. An exact $Z_{2}$ symmetry can be generated dynamically, e.g., as a remnant of a spontaneously broken $\mathrm{U}(1)$ gauge symmetry, such as $\mathrm{U}(1)_{B-L}[8-10]$. 
An attractive possibility is that $Z_{2}$, and consequently the DM stability, is an accidental symmetry. Examples include minimal DM [11, 12], hidden vector DM [13], and weakly interacting stable pions [14].

In this paper we explore a possibility that the discrete $Z_{2}$ that ensures the stability of DM is both accidental and approximate. As a result, the DM is metastable with decay times potentially close to the present observational bound of $\tau \gtrsim 10^{26} \mathrm{~s}$. We focus on a particular subset of asymmetric DM models [15] where DM carries baryon number. For recent reviews of asymmetric DM, see [16, 17]. Our working assumptions are

- Baryon number is a conserved quantum number (it could, for instance, be gauged at high scales).

- There is a sector that efficiently annihilates away the symmetric component. The exact form is not directly relevant for our discussion.

- The observed flavor structure in the quark sector is explained by flavor dynamics in the UV while DM is not charged under flavor.

The flavor dynamics fixes the flavor structure of dark sector couplings to the visible sector in the same way that it fixes the structure of the SM Yukawa interactions. This has two important consequences. First, the exchange of DM in the loops does not generate dangerously large Flavor Changing Neutral Currents (FCNCs). Secondly, and most importantly, a flavor singlet DM is stable on cosmological timescales even for $\mathrm{TeV}$ scale mediators between the dark and visible sectors. In this case, the nature of DM stability can even be probed directly at the LHC.

The underlying flavor symmetry is crucial for the stability of DM. We will demonstrate this for two realizations of flavor physics: the Minimal Flavor Violation (MFV) hypothesis and for abelian horizontal symmetries in the case where DM carries baryon number 2 . In this case the mediators leading to the decay of DM can be at $\mathcal{O}(100 \mathrm{GeV})$. In contrast, for completely anarchic flavor couplings where DM couples to all quark flavors with $\mathcal{O}(1)$ couplings, the indirect DM bounds would require the mediators to have masses in the $\mathcal{O}(10 \mathrm{TeV})$ range.

The implications of continuous flavor symmetries for DM interactions have also been explored in $[18 ?-28]$. Our analysis differs from these studies in that we are assuming that DM is a flavor singlet (as is the case in most models of DM). This, along with its small mass and conserved baryon number, also ensures that DM is metastable in our setup. The stability of symmetry-less DM in the context of discrete flavor groups has been discussed in [29] (for the potential relation of discrete flavor groups in the leptonic sector and the stability of DM, see also [30-32]). Furthermore, the stability of asymmetric DM due to a mirror baryon number was explored in [33] or due to fractional baryon number in [34]. The decaying DM in the context of ADM models was explored in [35-38].

The paper is structured as follows. In section 2, we review the relation between DM mass and relic abundance in asymmetric DM models. In section 3, we give two examples of flavor breaking models at the level of Effective Field Theory (EFT) analysis that can lead to metastable asymmetric DM. In section 4, we derive the indirect detection bounds 
on the two EFT set-ups. In section 5, we give two examples of mediators that would lead to the EFT set-ups discussed in section 4. The relevant bounds on the mediator masses and couplings, including collider signatures, are derived in section 6 . Conclusions are given in section 7 , while appendices contain technical details.

\section{Dark matter mass in asymmetric dark matter models}

Asymmetric Dark Matter (ADM) models [15, 39-52] address the question of why the DM density, $\Omega_{\chi}$, and the baryon density in the universe, $\Omega_{B}$, are so close to each other, $\Omega_{\chi} \simeq 5.3 \Omega_{\mathrm{B}}$ [53]. In the standard weakly interacting massive particle (WIMP) models of DM this is to some extent pure coincidence. In this case DM is a thermal relic and

$$
\left(\frac{\Omega_{\chi}}{0.265}\right)\left(\frac{h}{0.673}\right)^{2} \sim \frac{3 \times 10^{-27} \mathrm{~cm}^{3} \mathrm{~s}^{-1}}{\langle\sigma v\rangle}
$$

with $\langle\sigma v\rangle$ the thermally averaged DM annihilation cross section. The coincidence $\Omega_{\chi} \sim \Omega_{B}$ then arises due to a fortuitous size of the annihilation cross section for a weakly coupled weak scale DM - the WIMP miracle.

In contrast, in ADM models the observed DM is not a thermal relic. Its relic abundance reflects the asymmetry in DM, $\chi$, and anti-DM, $\chi^{\dagger}$, densities in the early universe. The $\chi$ and $\chi^{\dagger}$ annihilate away, and only the asymmetric component remains. The coincidence of $\Omega_{\chi}$ and $\Omega_{B}$ is then due to the fact that the DM relic abundance has the same origin as the baryon asymmetry. The difference between $\Omega_{\chi}$ and $\Omega_{\mathrm{B}}$ is simply due to the fact that the DM particle is more massive than a proton by a factor of a few. More precisely, to explain the observed $\Omega_{\chi}$ the DM's mass needs to be (see appendix B)

$$
m_{\chi}=N_{0} m_{p} \frac{\Omega_{\chi}}{\Omega_{B}} \frac{1}{(B-L)_{\chi}},
$$

where $m_{p}$ is the proton mass. Here $(B-L)_{\chi}$ is the $B-L$ charge of the $\chi$ field. The exact value of numerical prefactor $N_{0} \simeq \mathcal{O}(1)$ depends on when the operators transferring the baryon asymmetry between the visible and the dark sector decouple. For decoupling temperature above electroweak phase transition, and assuming that there are only the SM fields in the visible sector, gives $N_{0}=1.255$ for DM that is a complex scalar or a Dirac fermion. In this case the required DM mass is

$$
m_{\chi}=(6.2 \pm 0.4) \mathrm{GeV} \frac{1}{(B-L)_{\chi}},
$$

where the error reflects the errors on $\Omega_{\chi}=0.265 \pm 0.011$ and $\Omega_{B}=0.0499 \pm 0.0022[53,54]$. We thus have

$$
m_{\chi}=\{6.2,3.1,2.1\} \mathrm{GeV}, \quad \text { for } \quad(B-L)_{\chi}=\{1,2,3\},
$$

where we only quote the central values. Deviations from the above relations are possible if for instance the visible sector contains additional degrees of freedom beyond the SM. In that case, $m_{\chi}$ in $(2.2)$ is a function of $\left[(B-L)^{2}\right]_{\mathrm{NP}},\left[Y^{2}\right]_{N P}$, and $[Y(B-L)]_{N P}$, i.e., the 


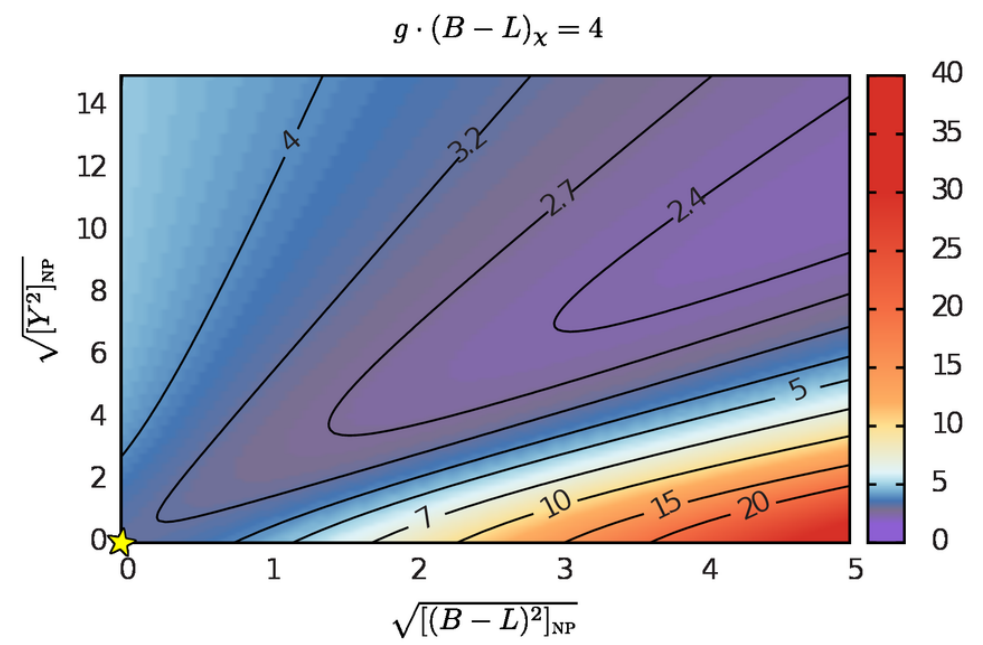

Figure 1. Contours of the ADM mass $m_{\chi}$ (in $\mathrm{GeV}$ ) required to obtain the correct DM relic density as a function of $\left[(B-L)^{2}\right]_{\mathrm{NP}},\left[Y^{2}\right]_{N P}$, i.e. the $(B-L)^{2}$, and $Y^{2}$ summed over effective d.o.f. in the visible NP sector, while keeping $[Y(B-L)]_{N P}=\sqrt{\left[(B-L)^{2}\right]_{\mathrm{NP}}\left[Y^{2}\right]_{N P}}$ and $g(B-L)_{\chi}=4$. The visible sector with only the $\mathrm{SM}$, eq. (2.2), is denoted by a star.

$(B-L)^{2}, Y^{2}$ and $Y(B-L)$ summed over effective degrees of freedom in the visible NP sector, cf. eq. (B.17). The $m_{\chi}$ required to obtain the correct relic abundance is shown in figure 1. For illustration, we set $[Y(B-L)]_{N P}=\sqrt{\left[(B-L)^{2}\right]_{\mathrm{NP}}\left[Y^{2}\right]_{N P}}$ in the plot and assume that DM is a complex scalar with $(B-L)_{\chi}=2$. We see that for $\left[(B-L)^{2}\right]_{\mathrm{NP}} \sim$ $\left[Y^{2}\right]_{N P} \sim[Y(B-L)]_{N P}$ the deviations from (2.4) are modest, of $\mathcal{O}(1)$. Further deviations from eqs. (2.2) and (2.4) are possible in more general frameworks such as ADM from leptogenesis [55] or dynamically induced mass mixing [56]. Henceforth, we will assume that $m_{\chi}$ is given by eqs. (2.2) and (2.4). Our results can be trivially adjusted if this is not the case.

For concreteness we assume in this paper the thermal history of the universe that closely resembles the one in [15] and has several distinct epochs relevant for the ADM relic density. At high temperatures, a $B-L$ asymmetry is generated, e.g., via GUT-like baryogenesis [15] or via leptogenesis [55]. The $B-L$ asymmetry is efficiently transferred between the visible and the DM sectors through asymmetric interactions. We do not require a discrete $Z_{n}$ symmetry in the dark sector so that, unlike [15], the asymmetric interactions can involve just a single $\chi$ field. At low energies, they have a schematic form

$$
\mathcal{O}_{\text {asymm. }} \sim \frac{C}{\Lambda^{6}} \chi(q q)^{3}
$$

taking $(B-L)_{\chi}=2$ complex scalar DM as an example. Here, $C$ is a flavor-dependent coefficient. The asymmetric interactions freeze out at temperature $T_{f} \sim \Lambda \gg m_{\chi}$, below which the $B-L$ asymmetries in the visible and dark sectors are separately conserved. If the flavor breaking is due to a spontaneously broken horizontal symmetry (see section 3.2), the freeze out temperature for the above dimension 10 operator in eq. (2.5) is, using Naive 
Dimensional Analysis (NDA),

$$
T_{f} \sim\left(1.66 \times \sqrt{g_{*}}\left(16 \pi^{2}\right)^{3} \frac{8 \pi}{C^{2}} \frac{\Lambda^{12}}{M_{\mathrm{Pl}}}\right)^{1 / 11} \simeq 480 \mathrm{GeV} .
$$

In the numerical evaluation, we used the lower bound $\Lambda=\Lambda_{*}=1.9 \mathrm{TeV}$ from indirect detection eq. (4.2), taken the effective number of relativistic d.o.f. to be $g_{*}=108.75$, corresponding to the SM with a complex scalar DM, and set $C=1$ which is appropriate for the $\chi b \rightarrow b s c t b$ transition dominance (with any permutation of the flavors). Note that $T_{f}$ is above the electroweak phase transition temperature $T_{\text {ew }} \sim 170 \mathrm{GeV}$. It is also well below $\Lambda$ so that the use of EFT is justified. If the mediator scale were too low, $\Lambda \lesssim 730 \mathrm{GeV}$ (or $\Lambda \lesssim 400 \mathrm{GeV}$ for MFV breaking), the asymmetric operator would not freeze out before electroweak phase transition started. Consequently, the DM quantum number would not be conserved and the DM density would be washed out. This places a lower bound on the asymmetric mediator masses to be above a few hundred $\mathrm{GeV}$.

Finally, at temperatures below the DM mass, the bulk of the DM efficiently annihilates back to the visible sector through symmetric interactions leaving only the small asymmetric component. We have nothing new to say about this mechanism and refer the reader to a set of model building ideas already present in the literature [16, 57-60].

\section{Metastability and flavor breaking}

We show next that the DM in ADM models can be stable on cosmological time-scales without invoking discrete $Z_{n}$ symmetries. We assume that the SM quark flavor structure is explained by a continuous flavor group and that the DM carries nonzero baryon number. This is a crucial ingredient in the argument. Since DM is not charged under the flavor group, while the SM fields are, there are no interactions between DM and the SM in the limit that the flavor group is unbroken (all flavor singlet interactions are forbidden by baryon number conservation). All the interactions between DM and the visible sector thus have to be flavor breaking and this leads to a significant suppression of the DM decay time.

We show this explicitly for two examples of flavor breaking: i) the MFV ansatz, where all the flavor breaking is assumed to be due to the SM Yukawas, and ii) the spontaneously broken horizontal U(1) symmetries. Integrating out the NP fields gives the effective DM decay Lagrangian

$$
\mathcal{L}=\sum_{i} \frac{C_{i}}{\Lambda^{\left(D_{i}-4\right)}} \mathcal{O}_{i}
$$

The sizes of the Wilson coefficients, $\mathcal{C}_{i}$, are fixed by the assumed flavor generating mechanism. We consider the case of DM, $\chi$, that is a SM gauge singlet but carries nonzero baryon number, $B \neq 0$. The lowest dimensional asymmetric local operators thus have the generic form

$$
\mathcal{O}_{i}=\chi\left[u^{c}\right]^{n_{u}}\left[d^{c}\right]^{n_{d}}\left[q^{*}\right]^{n_{q}},
$$

where we do not show the contractions of SM gauge indices. Here $\left(n_{u}+n_{d}+n_{q}\right) \bmod 3=0$ since DM is a color singlet. Note that DM needs to carry an integer baryon number in 
order not to forbid all the asymmetric interactions with the visible sector. Above, $u^{c}, d^{c}$ are the electroweak singlets and $q$ represents the electroweak doublet left-handed quark fields in two component notation, with $q^{*}$ being the corresponding complex conjugated Weyl spinor, see appendix A. In the down-quark mass basis they are

$$
u^{c} \rightarrow u_{\mathrm{MASS}}^{c}, \quad d^{c} \rightarrow d_{\mathrm{MASS}}^{c}, \quad q=\left(\begin{array}{l}
u \\
d
\end{array}\right) \rightarrow\left(\begin{array}{c}
V_{\mathrm{CKM}} u_{\mathrm{MASS}} \\
d_{\mathrm{MASS}}
\end{array}\right) .
$$

The SM Yukawa matrices are then

$$
Y_{D} \rightarrow Y_{D}^{\text {diag }}, \quad Y_{U} \rightarrow V_{\mathrm{CKM}} Y_{U}^{\text {diag }}
$$

with $Y_{D, U}^{\text {diag }}$ the diagonal Yukawa matrices.

As an example, let us consider fermionic $B=1 \mathrm{DM}$. Two distinct types of operators are allowed

$$
\begin{aligned}
& \mathcal{O}_{1}^{(B=1)}=\left(\chi u^{c}\right)\left(d^{c} d^{c}\right) \rightarrow\left(\chi u_{\mathrm{MASS}}^{c}\right)\left(d_{\mathrm{MASS}}^{c} d_{\mathrm{MASS}}^{c}\right), \\
& \mathcal{O}_{2}^{(B=1)}=\left(\chi q_{\rho}^{*}\right)\left(d^{c} q_{\sigma}^{*}\right) \epsilon^{\rho \sigma} \rightarrow\left(\chi u_{\mathrm{MASS}}^{*} V_{\mathrm{CKM}}\right)\left(d_{\mathrm{MASS}}^{c} d_{\mathrm{MASS}}^{*}\right),
\end{aligned}
$$

where $\rho, \sigma$ are $\mathrm{SU}(2)_{L}$ indices while the $\mathrm{SU}(3)_{C}$ and flavor indices are implicit and we have chosen one possible Lorentz contraction denoted by the parentheses.

\subsection{Minimal Flavor Violation}

The MFV assumption is that, also in the NP sector, the flavor is broken only by the SM Yukawas $Y_{U, D}$ [61-65]. The MFV assumption can be most succinctly cast in the spurion language [62]. In the limit of vanishing quark masses the SM quark sector enjoys an enhanced flavor symmetry $G_{F}=\mathrm{SU}(3)_{Q} \times \mathrm{SU}(3)_{U} \times \mathrm{SU}(3)_{D}$. The Yukawa interactions $u^{c} Y_{U}^{\dagger} q H, d^{c} Y_{D}^{\dagger} q H^{c}$ are formally invariant under $G_{F}$, if $Y_{U, D}$ are promoted to spurions, i.e. if they are assumed to transform under $G_{F}$ as $Y_{U} \rightarrow Y_{U}^{\prime}=U_{Q} Y_{U} U_{U}^{\dagger}, Y_{D} \rightarrow Y_{D}^{\prime}=U_{Q} Y_{D} U_{D}^{\dagger}$. Here $U_{Q, U, D}$ are transformations from $\mathrm{SU}(3)_{Q, U, D}$, respectively.

This means that the low energy operators in (3.1) also need to be formally $G_{F}$ invariant. Keeping only the minimal insertion of Yukawas, the operators $\mathcal{O}_{1,2}$ in eq. (3.5) for $B=1$ DM are

$$
\begin{aligned}
& \mathcal{O}_{1}^{(B=1)}=\left(\chi u_{\alpha}^{c} Y_{U}^{\dagger} Y_{D}\right)_{K}\left(d_{N \beta}^{c} d_{M \gamma}^{c}\right) \epsilon^{K N M} \epsilon^{\alpha \beta \gamma} \\
& \rightarrow\left(\chi u_{\mathrm{MASS}}^{c} Y_{U}^{\text {diag } \dagger} V_{\mathrm{CKM}}^{\dagger} Y_{D}^{\text {diag }}\right)_{K \alpha}\left(\left[d_{\mathrm{MASS}}^{c}\right]_{N \beta}\left[d_{\mathrm{MASS}}^{c}\right]_{M \gamma}\right) \epsilon^{K N M} \epsilon^{\alpha \beta \gamma}, \\
& \mathcal{O}_{2}^{(B=1)}=\left(\chi q_{K \alpha i}^{*}\right)\left(\left[d_{\beta}^{c} Y_{D}^{\dagger}\right]_{N} q_{M \gamma j}^{*}\right) \epsilon^{i j} \epsilon^{K N M} \epsilon^{\alpha \beta \gamma} \\
& \rightarrow\left(\chi u_{\mathrm{MASS}}^{*} V_{\mathrm{CKM}}^{\dagger}\right)_{K \alpha}\left(\left[d_{\mathrm{MASS}}^{c} Y_{D}^{\text {diag } \dagger}\right]_{N \beta}\left[d_{\mathrm{MASS}}^{*}\right]_{M \gamma}\right) \epsilon^{K N M} \epsilon^{\alpha \beta \gamma},
\end{aligned}
$$

where $\alpha, \beta, \gamma$ are the color indices, and $K, N, M$ run over the quark generations.

The two operators lead to the $\chi \rightarrow$ bus decay at the partonic level which is the least suppressed kinematically allowed transition. For the operator $\mathcal{O}_{1}$, this transition arises at 1-loop and requires two chirality flips, see figure 2. The decay amplitude scales as $\sim y_{t} y_{b}$ with an extra loop factor and a chirality flip suppression $\sim m_{t} \Lambda_{\mathrm{QCD}} / m_{W}^{2}$. To be conservative, we count the chirality flip suppression due to the light $u, d, s$ quarks as 


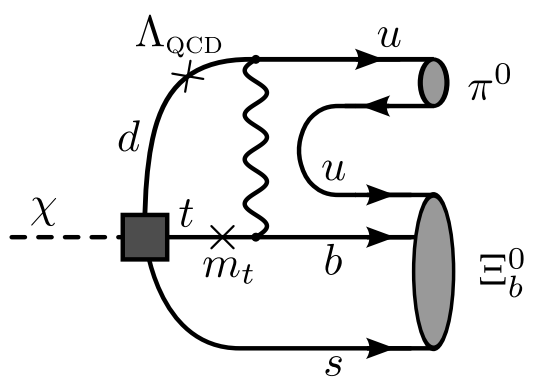

Figure 2. Feynman diagram for the decay of DM with $B=1$ assuming MFV. This amplitude leads to the partial decay width $\Gamma_{\chi}^{(1)}$ in eq. (3.7).

proportional to $\Lambda_{\mathrm{QCD}}$ and not to the much smaller quark masses. The operator $\mathcal{O}_{2}$ leads to the decay $\chi \rightarrow$ bus at tree level with the decay amplitude suppressed by $\sim y_{b} V_{u b}$. Once the quarks hadronize, the decays appear as $\chi \rightarrow \Xi_{b} \pi$, or $\chi \rightarrow \Lambda_{b} K$, with any number of pions. Using NDA to estimate the decay width gives ( $\operatorname{setting} V_{t b} \simeq V_{u d} \simeq 1$ )

$$
\begin{aligned}
& \Gamma_{\chi}^{(1)} \sim \frac{\left(y_{t} y_{b}\right)^{2}}{8 \pi}\left(\frac{m_{\chi}}{\Lambda}\right)^{4}\left(\frac{1}{16 \pi^{2}} \frac{m_{t} \Lambda_{\mathrm{QCD}}}{m_{W}^{2}}\right)^{2} \frac{m_{\chi}}{16 \pi^{2}}=6.6 \cdot 10^{-51} \mathrm{GeV}\left(\frac{y_{b}}{0.024}\right)^{2}\left(\frac{4.0 \cdot 10^{6} \mathrm{TeV}}{\Lambda}\right)^{4}, \\
& \Gamma_{\chi}^{(2)} \sim \frac{\left|y_{b} V_{u b}\right|^{2}}{8 \pi}\left(\frac{m_{\chi}}{\Lambda}\right)^{4} \frac{m_{\chi}}{16 \pi^{2}}=6.6 \cdot 10^{-51} \mathrm{GeV}\left(\frac{y_{b}}{0.024}\right)^{2}\left(\frac{4.3 \cdot 10^{7} \mathrm{TeV}}{\Lambda}\right)^{4},
\end{aligned}
$$

for the case where $\mathcal{O}_{1}$ and $\mathcal{O}_{2}$ dominate the decay, respectively. The last $1 / 16 \pi^{2}$ factor is due to three body final state and is required to obtain the correct estimate for the inclusive decay width as can be seen from the optical theorem and the use of the OPE. In the numerics, we use $m_{t}=173 \mathrm{GeV}, m_{\chi}=6.2 \mathrm{GeV},\left|V_{u b}\right|=0.00415$. The numerical prefactor $6.6 \cdot 10^{-51} \mathrm{GeV}=1 /\left(10^{26} s\right)$ is chosen to make contact with the bounds on the DM lifetime from indirect DM searches.

Note that MFV leads to two sources of suppression. First, there is the suppression of the Wilson coefficients due to Yukawa insertions, $y_{b} \sim 0.024$ for $\mathcal{O}_{1}$ and $y_{b} V_{u b} \sim 10^{-4}$ for $\mathcal{O}_{2}$. In addition, there is a loop suppression for $\mathcal{O}_{1}$ where the decay has to proceed through an off-shell top quark. Without these additional suppressions, the bounds from indirect DM detection would require about two orders larger NP scale, $\Lambda \gtrsim 4.3 \cdot 10^{9} \mathrm{TeV}$.

The suppression factors are much larger for $B=2 \mathrm{DM}$, in which case the DM is a scalar, and the asymmetric operators start at dimension 10 . We investigate in detail the operator

$$
\begin{aligned}
\mathcal{O}_{1}^{(B=2)}=\chi\left(d_{K \alpha}^{c} d_{N \beta}^{c}\right) & \left(\left[q^{*} Y_{D}\right]_{M \alpha^{\prime}} q_{K^{\prime} \beta^{\prime}}^{*}\right)\left(q_{N^{\prime} \gamma^{\prime}}^{*} q_{M^{\prime} \gamma}^{*}\right) \epsilon^{K N M} \epsilon^{K^{\prime} N^{\prime} M^{\prime}} \epsilon^{\alpha \beta \gamma} \epsilon^{\alpha^{\prime} \beta^{\prime} \gamma^{\prime}} \\
\rightarrow \chi & \left(\left[d_{\mathrm{MASS}}^{c}\right]_{K \alpha}\left[d_{\mathrm{MASS}}^{c}\right]_{N \beta}\right)\left(\left[u_{\mathrm{MASS}}^{*} V_{\mathrm{CKM}}^{\dagger} Y_{D}^{\mathrm{diag}}\right]_{M \alpha^{\prime}}\left[d_{\mathrm{MASS}}^{*}\right]_{K^{\prime} \beta^{\prime}}\right) \\
& \times\left(\left[u_{\mathrm{MASS}}^{*} V_{\mathrm{CKM}}^{\dagger}\right]_{N^{\prime} \gamma^{\prime}}\left[d_{\mathrm{MASS}}^{*}\right]_{M^{\prime} \gamma}\right) \epsilon^{K N M} \epsilon^{K^{\prime} N^{\prime} M^{\prime}} \epsilon^{\alpha \beta \gamma} \epsilon^{\alpha^{\prime} \beta^{\prime} \gamma^{\prime}},
\end{aligned}
$$

that gives the least suppressed decay amplitude. Above, we chose one of the possible color contractions, implicitly assumed contractions of weak indices within brackets, and only kept the weak contraction leading to the largest decay rate in the second line. 


\begin{tabular}{|ccccccccc|}
\hline \multicolumn{4}{c}{ ADM model } & \multicolumn{3}{c}{ MFV } & \multicolumn{3}{c|}{ FN } \\
$B$ & Dim. & $m_{\chi}[\mathrm{GeV}]$ & decay & $\tau[\mathrm{s}]$ & $\Lambda[\mathrm{TeV}]$ & decay & $\tau[\mathrm{s}]$ & $\Lambda[\mathrm{TeV}]$ \\
\hline 1 & 6 & 6.2 & $\chi \rightarrow$ bus & $10^{26}$ & $4.0 \times 10^{6}$ & $\chi \rightarrow$ bus & $10^{26}$ & $8.1 \times 10^{8}$ \\
2 & 10 & 3.1 & $\chi \rightarrow$ udsuds & $10^{26}$ & 0.63 & $\chi \rightarrow$ udsuds & $10^{26}$ & 2.5 \\
3 & 15 & 2.1 & forbidden & $\infty$ & - & forbidden & $\infty$ & - \\
\hline
\end{tabular}

Table 1. Leading decay modes for the $B=1,2,3$ ADM assuming MFV or FN flavor breaking. The dimensionality of the decay operators are denoted in the 2 nd column. With the suppression scales $\Lambda$ given in the 6 th and 9 th column the ADM decay time is $\tau \simeq 10^{26} \mathrm{~s}$. The $B=3$ ADM decays to quarks are kinematically forbidden.

The correct relic abundance requires a DM mass of $m_{\chi}=3.1 \pm 0.2 \mathrm{GeV}$, assuming the SM field content at the time of the decoupling of the asymmetric operators. We assume that $m_{\chi}<m_{\Lambda_{c}^{+}}+m_{\Sigma^{-}}=3.48 \mathrm{GeV}$, and thus below the threshold for the $\chi \rightarrow \Lambda_{c}^{+} \Sigma^{-}$ decay, kinematically forbidding the $\chi \rightarrow u d c d d s$ partonic transition. The least suppressed partonic level transition is therefore $\chi \rightarrow u d s u d s$ resulting, after hadronization, in the decays $\chi \rightarrow \Lambda^{0} \Lambda^{0}, \Sigma^{-} \Sigma^{+}, \Xi^{-} p, \Xi^{0} n, \ldots$ The NDA estimate of the $\chi$ decay width is then

$$
\Gamma_{\chi}^{(1)} \sim \frac{\left|y_{b} V_{u b}^{2}\right|^{2}}{8 \pi}\left(\frac{m_{\chi}}{\Lambda}\right)^{12} \frac{m_{\chi}}{\left(16 \pi^{2}\right)^{4}}=6.6 \cdot 10^{-51} \mathrm{GeV}\left(\frac{y_{b}}{0.024}\right)^{2}\left(\frac{0.63 \mathrm{TeV}}{\Lambda}\right)^{12} .
$$

The MFV assumption results in the $y_{b} V_{u b}^{2}$ suppression of the Wilson coefficient. The $1 /\left(16 \pi^{2}\right)^{4}$ factor reflects the fact that, in the OPE, the leading contribution starts at 5 loops. The use of the OPE may be suspect for such low $m_{\chi}$ masses and one could expect $\mathcal{O}(1)$ corrections to the above estimate from additional soft gluon loops.

Indirect DM searches require the NP scale to be $\Lambda \gtrsim 0.49 \mathrm{TeV}$. This corresponds to the bounds on the masses of the mediators between the dark and the visible sectors, $m_{\text {mediator }} \gtrsim 490 \mathrm{GeV}, m_{\text {mediator }} \gtrsim 210 \mathrm{GeV}$, and $m_{\text {mediator }} \gtrsim 90 \mathrm{GeV}$, if the operator (3.8) arises at tree level, 1-loop, or 2-loops, respectively. The mediators can thus be searched for at the LHC as discussed in section 6.3. Note that the flavor suppression was essential to have such a low bound on the NP scale $\Lambda$. Without it, and taking the Wilson coefficient to be 1 , the indirect bounds on the stability of $\mathrm{DM}$ would require $\Lambda \gtrsim 7.3 \mathrm{TeV}$, implying that the mediators were most likely out of reach of the LHC.

The bound on the NP scale $\Lambda$ is quite sensitive to the actual value of $m_{\chi}$. For larger values of $m_{\chi}$, the $\chi$ can decay to top and bottom quarks reducing the loop and CKM suppression of the decay width. This is illustrated in figure 3, where the NP scale is fixed to $\Lambda_{\mathrm{MFV}}=1 \mathrm{TeV}$ and $m_{\chi}$ is varied. As the kinematic thresholds for the $\chi$ decays to $c$ or $b$ quarks are reached, this results in a change of several orders of magnitude in the predicted decay time.

\subsection{Spontaneously broken horizontal symmetries}

The suppression we found above using the MFV ansatz is model dependent. To illustrate this point we turn to U(1) Frogatt-Nielsen (FN) models of spontaneously broken horizontal symmetries [66]. The suppression of the Wilson coefficients in the effective Lagrangian (3.1) 
is then given by the horizontal charges of the quarks in the operators. For instance, for the two $B=1$ DM operators in (3.1)

$$
\begin{aligned}
& \mathcal{O}_{1}^{(B=1)}=\left(\chi d_{K}^{c}\right)\left(u_{N}^{c} d_{M}^{c}\right) \rightarrow\left(\chi\left[d_{\mathrm{MASS}}^{c}\right]_{K}\right)\left(\left[u_{\mathrm{MASS}}^{c}\right]_{N}\left[d_{\mathrm{MASS}}^{c}\right]_{M}\right), \\
& \mathcal{O}_{2}^{(B=1)}=\left(\chi q_{K i}^{*}\right)\left(d_{N}^{c} q_{M j}^{*}\right) \epsilon^{i j} \rightarrow\left(\chi\left[u_{\mathrm{MASS}}^{*}\right]_{K}\right)\left(\left[d_{\mathrm{MASS}}^{c}\right]_{N}\left[d_{\mathrm{MASS}}^{*}\right]_{M}\right),
\end{aligned}
$$

the Wilson coefficients are

$$
C_{1} \sim \lambda^{\left|H\left(d_{K}^{c}\right)+H\left(u_{N}^{c}\right)+H\left(d_{M}^{c}\right)\right|}, \quad C_{2} \sim \lambda^{\left|-H\left(q_{K}\right)+H\left(d_{N}^{c}\right)-H\left(q_{M}\right)\right|} .
$$

Here $H\left(u_{K}^{c}\right), \ldots$, with $H\left(q_{K}^{*}\right)=-H\left(q_{K}\right)$, are the horizontal $\mathrm{U}(1)$ charges of the quarks, and $\lambda \sim 0.2$ is the expansion parameter. The dependence of the operators and Wilson coefficients on the generational indices $K N M$ is implicit as are color, weak, and Lorentz contractions in (3.10).

An example of a horizontal charge assignment that gives phenomenologically satisfactory quark masses and CKM matrix elements is [67],

$$
\begin{aligned}
& \begin{array}{lll}
1 & 2 & 3
\end{array} \\
& H\left(q, d^{c}, u^{c}\right) \Rightarrow \begin{array}{l}
q \\
d^{c} \\
u^{c}
\end{array}\left(\begin{array}{lll}
3 & 2 & 0 \\
3 & 2 & 2 \\
3 & 1 & 0
\end{array}\right),
\end{aligned}
$$

where the column labels $\{1,2,3\}$ correspond to the first, second, and third generations of quarks.

Since the heavier flavors carry smaller charges the DM preferentially decays into the heaviest accessible states. As in MFV, the dominant decay is $\chi \rightarrow b u s$, except that the $y_{b} V_{u b} \sim \lambda^{5}$ suppression gets replaced by a much more modest $\sim \lambda^{\left|-H\left(q_{1}\right)+H\left(s^{c}\right)-H\left(q_{3}\right)\right|}=\lambda$. This is the largest scaling allowed by FN charges. In concrete UV mediator models the suppression can, in fact, be much more severe as we will see explicitly in the next section.

For $B=2 \mathrm{DM}$ the least suppressed operator is

$$
\begin{aligned}
\mathcal{O}_{1}^{(B=2)}=\chi\left(d_{K}^{c} d_{N}^{c}\right)\left(q_{M}^{*} q_{K^{\prime}}^{*}\right)\left(q_{N^{\prime}}^{*} q_{M^{\prime}}^{*}\right) & \\
\rightarrow & \chi\left(\left[d_{\text {MASS }}^{c}\right]_{K}\left[d_{\text {MASS }}^{c}\right]_{N}\right)\left(\left[u_{\text {MASS }}^{*}\right]_{M}\left[d_{\text {MASS }}^{*}\right]_{K^{\prime}}\right)\left(\left[u_{\text {MASS }}^{*}\right]_{N^{\prime}}\left[d_{\text {MASS }}^{*}\right]_{M^{\prime}}\right),
\end{aligned}
$$

suppressing, again, the color and weak contractions. The corresponding Wilson coefficient is suppressed by

$$
C_{1} \sim \lambda^{\left|H\left(d_{K}^{c}\right)+H\left(d_{N}^{c}\right)-H\left(q_{M}\right)-H\left(q_{K^{\prime}}\right)-H\left(q_{N^{\prime}}\right)-H\left(q_{M^{\prime}}\right)\right|} .
$$

At the partonic level, the dominant decay is $\chi \rightarrow$ uss uds with a Wilson coefficient that is of parametric size $\sim \lambda^{\left|H\left(d^{c}\right)+H\left(s^{c}\right)-2 H\left(q_{2}\right)-2 H\left(q_{1}\right)\right|}=\lambda^{5}$. Note that in MFV this process proceeded through 2 loops so that the suppression was much more severe, $\sim V_{t s} V_{u b} /\left(16 \pi^{2}\right)^{2} \sim$ $\lambda^{5} /\left(16 \pi^{2}\right)^{2}$ at the amplitude level. While the suppression in the FN case is much less than in the MFV case, it is still nontrivial. It lowers the scale of NP allowed by indirect DM searches from $\Lambda \gtrsim 7.3 \mathrm{TeV}$, in the case of no flavor structure, to $\Lambda \gtrsim 2.5 \mathrm{TeV}$ in the FN case. Taking the bound from DM indirect detection searches gives $\Lambda \gtrsim 1.9 \mathrm{TeV}$. If the operator arises at tree level, 1-loop or 2-loops, this corresponds to mediator masses, $m_{\text {mediator }} \gtrsim 1.9 \mathrm{TeV}, m_{\text {mediator }} \gtrsim 830 \mathrm{GeV}$, and $m_{\text {mediator }} \gtrsim 360 \mathrm{GeV}$, respectively. 


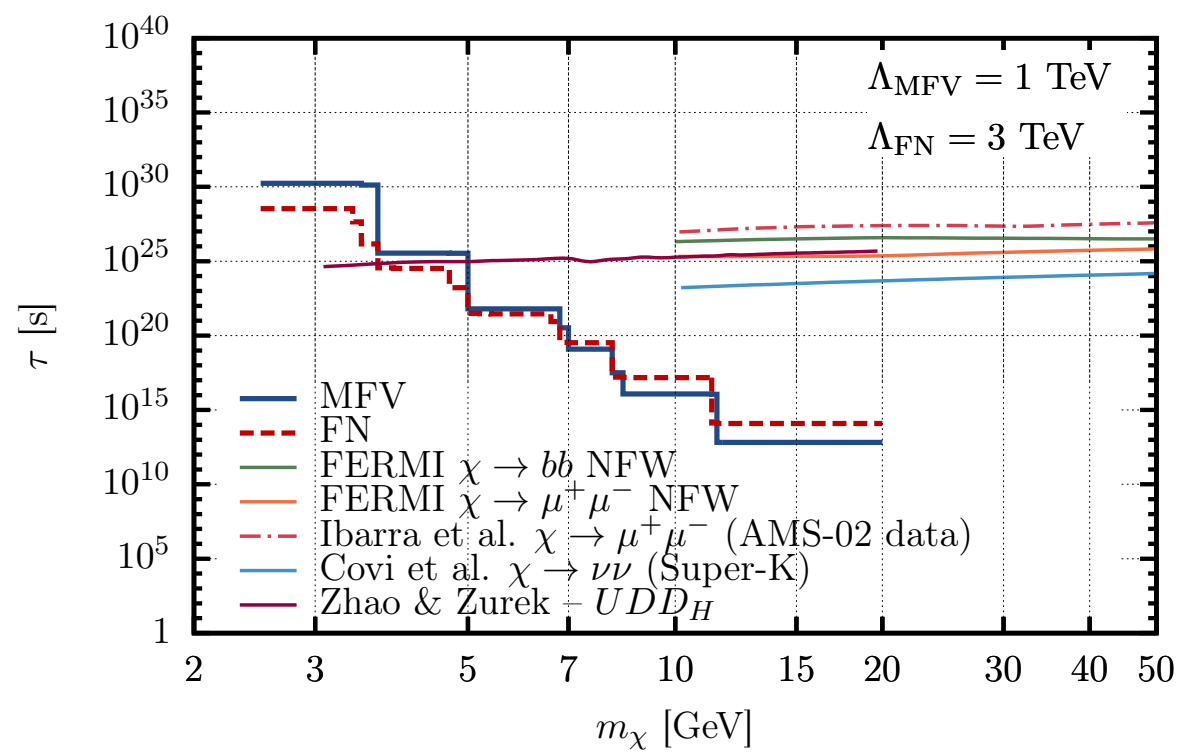

Figure 3. The solid blue (red dashed) line denotes the $B=2 \mathrm{DM}$ lifetime as a function of $m_{\chi}$ for the MFV (FN) case, fixing the NP scale to $\Lambda=1(3) \mathrm{TeV}$. Assuming the dominance of one decay mode, the green (orange) line shows the constraint on the decay time from FERMI-LAT [68] for $b \bar{b}\left(\mu^{+} \mu^{-}\right)$final states using the NFW profile. The dash-dotted red line shows the AMS-02 [69] constraint on $\chi \rightarrow \mu^{+} \mu^{-}$decay time derived in [70], while the light blue line shows the SuperKamiokande [71] constraint on the $\chi \rightarrow \nu \bar{\nu}$ decay time obtained in [72]. The purple line shows the upper limit on $\chi \rightarrow u d s$ and $\chi \rightarrow c b s$ decay times (indistinguishable at the scale of the figure) obtained in [35].

\section{Indirect detection}

The asymmetric operators discussed in the previous section lead to a decaying DM which can be potentially seen in indirect DM searches. In our models, the $\chi$ decays hadronicaly. The decay products thus contain a number of charged particles and photons. The flavor composition of the final state depends on the mass, $m_{\chi}$, and also on the assumed flavor breaking pattern. In section 3, we discussed in detail the case of $6.2 \mathrm{GeV} B=1 \mathrm{DM}$, which decays through $\chi \rightarrow$ bus and a $3.1 \mathrm{GeV} B=2 \mathrm{DM}$ that decays through $\chi \rightarrow$ uds uds. After hadronization, these result in the decays $\chi \rightarrow \Xi_{b}^{0} \pi^{0}$ and $\chi \rightarrow \Lambda^{0} \Lambda^{0}$, respectively. The dominant decays for other DM masses, assuming the MFV or FN flavor breaking patterns, are given in appendix C. The DM lifetime dependence on $m_{\chi}$ is shown in figure 3 after fixing the NP scale to be $\Lambda=1(3) \mathrm{TeV}$ for the MFV (FN) flavor breaking.

To guide the eye, we also show in figure 3 the following bounds from indirect DM searches. The green (orange) line shows the constraint on the DM decay time from FERMILAT [68] for $\chi \rightarrow b \bar{b}\left(\mu^{+} \mu^{-}\right)$decays using the NFW profile. The dash-dotted light red line shows the results of an analysis [70] based on AMS-02 [69] and assuming $\chi \rightarrow \mu^{+} \mu^{-}$. The light blue line shows the result of an analysis [72] assuming $\chi \rightarrow \bar{\nu} \nu$ decay based on Super-Kamiokande [71] bounds. The purple line is an exclusion curve from [35] based on galactic and extragalactic gamma ray flux measurements by Fermi [73-75]. The authors 


\begin{tabular}{|cccccc|}
\hline Field & $\mathrm{SU}(3)_{C}$ & $\mathrm{SU}(2)_{L}$ & $\mathrm{U}(1)_{Y}$ & $G_{F}$ & $\mathrm{U}(1)_{B-L}$ \\
\hline$\phi_{L}$ & $\overline{\mathbf{3}}$ & $\mathbf{1}$ & $1 / 3$ & $(\mathbf{6}, \mathbf{1}, \mathbf{1})$ & $2 / 3$ \\
$\varphi_{L}$ & $\mathbf{6}$ & $\mathbf{1}$ & $1 / 3$ & $(\overline{\mathbf{3}}, \mathbf{1}, \mathbf{1})$ & $2 / 3$ \\
$\phi_{R}$ & $\overline{\mathbf{3}}$ & $\mathbf{1}$ & $-2 / 3$ & $(\overline{\mathbf{3}}, \mathbf{1}, \mathbf{1})$ & $2 / 3$ \\
\hline
\end{tabular}

Table 2. The gauge and global charge assignment for the three scalar mediators, $\phi_{L}, \varphi_{L}$ and $\phi_{R}$, in the first UV completion toy model for which we assume the MFV flavor breaking pattern.

in [35] consider $\chi \rightarrow u d s$ and $\chi \rightarrow c b s$ decays as two extreme choices for the flavor structure of the final states. The derived bounds on the $\chi$ lifetime differ by less then a factor of 2 such that the two bounds overlap on the scale of figure 3. The decays we consider fall between these two extreme choices with potentially weakened bounds in our cases above $m_{\chi} \gtrsim \mathcal{O}(10) \mathrm{GeV}$ due to the increased multiplicity of final states. The bounds cross the expected $\chi$ decay times at $m_{\chi} \sim 5 \mathrm{GeV}$ for $\Lambda_{\mathrm{MFV}}=1 \mathrm{TeV}$ suppression scale in the case of MFV flavor breaking and at $m_{\chi} \sim 4 \mathrm{GeV}$ for $\Lambda_{\mathrm{FN}}=3 \mathrm{TeV}$ suppression scale in the case of FN flavor breaking.

For the $3.1 \mathrm{GeV} B=2 \mathrm{DM}$, we thus find that, for the MFV case, the indirect detection requires

$$
\Lambda_{\mathrm{MFV}} \gtrsim 0.49 \mathrm{TeV},
$$

where the dominant operator is given in (3.8). For the FN case the bound is

$$
\Lambda_{\mathrm{FN}} \gtrsim 1.9 \mathrm{TeV}
$$

where the least suppressed operator is given in (3.13).

\section{Mediator models}

The EFT analysis of metastable ADM using asymmetric operators is an appropriate approach to derive the indirect DM detection signatures as we did in the previous section. However, for DM direct detection searches and the DM production at colliders, the dominant signals are due to either a single mediator exchange or from direct production of the mediators. To assess the reach of these DM searches, the UV completions to our models are therefore needed.

We introduce two toy model UV completions that can generate the dimension 10 effective operators; that is, the operator in eq. (3.8) for the MFV case and the operator in eq. (3.13) for the FN case. The EFT operators are generated when the $\sim \mathrm{TeV}$ mediators are integrated out. In our first model, all the mediators are scalars, while in the second model there is also a fermionic mediator. The flavor structure in either of the two models could be of the MFV or of the FN type. For concreteness we fix the first model to have the MFV flavor breaking, and the second model to have the FN flavor breaking.

\subsection{MFV model with scalar mediators}

The SM is extended by the DM, $\chi$, and three flavor multiplets of scalar mediators - a color anti-triplet $\phi_{L}$ and a color sextet $\varphi_{L}$, both with hypercharge $1 / 3$, and a color sextet $\phi_{R}$ with 


\begin{tabular}{|ccccc|}
\hline Field & $\mathrm{SU}(3)_{C}$ & $\mathrm{SU}(2)_{L}$ & $\mathrm{U}(1)_{Y}$ & $\mathrm{U}(1)_{B-L}$ \\
\hline$\phi$ & $\overline{\mathbf{3}}$ & $\mathbf{1}$ & $1 / 3$ & $2 / 3$ \\
$\psi$ & $\mathbf{1}$ & $\mathbf{1}$ & 0 & 1 \\
\hline
\end{tabular}

Table 3. Gauge and $B-L$ charges of the mediators $\phi$ and $\psi$ in the second UV completion toy model. We also assume the FN flavor breaking pattern.

hypercharge $-2 / 3$ (see table 2). They transform under the flavor group $G_{F}$ as $(\mathbf{6}, \mathbf{1}, \mathbf{1})$, $(\overline{\mathbf{3}}, \mathbf{1}, \mathbf{1})$, and $(\overline{\mathbf{3}}, \mathbf{1}, \mathbf{1})$, respectively. The interaction Lagrangian between mediators and the $\mathrm{SM}$ is thus given by

$$
\begin{aligned}
\mathcal{L}_{\mathrm{INT}} & \supset \frac{\kappa_{1}}{2} \bar{K}_{I}^{A B}\left[\phi_{L}\right]_{\gamma}^{I}\left(q_{A, \alpha i}^{*} q_{B, \beta j}^{*}\right) \epsilon^{i j} \epsilon^{\alpha \beta \gamma}+\frac{\kappa_{2}}{2} \bar{K}_{\lambda}^{\alpha \beta}\left[\varphi_{L}\right]_{A}^{\lambda}\left(q_{B, \alpha i}^{*} q_{C, \beta j}^{*}\right) \epsilon^{i j} \epsilon^{A B C} \\
& +\frac{\kappa_{3}}{2}\left[Y_{D}\right]_{X}^{A}\left[\phi_{R}\right]_{A, \alpha}\left(d_{Y, \beta}^{c} d_{Z, \gamma}^{c}\right) \epsilon^{\alpha \beta \gamma} \epsilon^{X Y Z}+\kappa_{4} \bar{K}_{I}^{A B} \bar{K}_{\lambda}^{\alpha \beta} \chi^{\dagger}\left[\phi_{L}\right]_{\alpha}^{I}\left[\varphi_{L}\right]_{A}^{\lambda}\left[\phi_{R}\right]_{B, \beta}+\text { h.c. },
\end{aligned}
$$

where the flavor indices $A, B, C$ belong to $\mathrm{SU}(3)_{Q}$ and $X, Y, Z$ to $\mathrm{SU}(3)_{D}$. The QCD indices are $\alpha \beta \gamma$, while the weak isospin indices are denoted by $i, j$. The flavor index $I$ and color index $\lambda$ run from 1 to 6 . The matrices of the Clebsch-Gordan coefficients, $\bar{K}_{I}^{A B}$ and $\bar{K}_{\lambda}^{\alpha \beta}$, are the same as in [76] and satisfy the completeness relation $\left(\bar{K}_{I}^{A B}\right)^{*} \bar{K}_{I}^{C D}=$ $\frac{1}{2}\left(\delta_{A}^{D} \delta_{B}^{C}+\delta_{A}^{C} \delta_{B}^{D}\right)$, and similary for $\bar{K}_{\lambda}^{\alpha \beta}$. In the second line of (5.1), the down Yukawa insertions make the interaction term with right-handed down quarks formally invariant under $G_{F}$.

Integrating out the mediators $\phi_{L, R}, \varphi_{L}$, gives the $\chi$ decay operator (3.8), with the Wilson coefficient

$$
\frac{\mathcal{C}_{1}}{\Lambda^{6}}=-\frac{1}{8} \frac{\kappa_{1} \kappa_{2} \kappa_{3} \kappa_{4}}{m_{\phi_{L}}^{2} m_{\varphi_{L}}^{2} m_{\phi_{R}}^{2}} .
$$

For $\kappa_{1}=\kappa_{2}=\kappa_{3}=\kappa_{4}=1$ the bounds from indirect DM searches thus require $m_{\phi_{L}, \phi_{R}, \varphi_{L}} \gtrsim 450 \mathrm{GeV}$, if all the mediator masses are the same. This should be appropriately rescaled if either $\kappa_{i}$ have smaller values or if all masses are not the same. For instance, for $\kappa_{i}=0.3$ the mass degenerate case of the mediators is bounded from below by $m_{\phi_{L}, \phi_{R}, \varphi_{L}} \gtrsim$ $200 \mathrm{GeV}$. Since the mediators carry color charges, they can be searched for at the LHC as discussed in section 6.3 below.

Note that, for the Lagrangian in eq. (5.1) the common scenario where the symmetric component of $\chi$ density annihilates through a dark photon [16,57-59] is phenomenologically not viable. In this case, at least some of the SM quark fields would need to carry a dark $\mathrm{U}(1)$ charge in conflict with the low energy constraints if dark photon is light. A viable possibility, on the other hand, is the annihilation of $\chi \chi^{\dagger}$ to a pair of light scalars along the lines of ref. [77].

\subsection{FN model with fermionic and scalar mediators}

In the second model the SM is supplemented with a DM scalar $\chi$, a Dirac fermion $\psi$ and a complex scalar $\phi$ with SM gauge assignments as in table 3. The relevant terms in the 

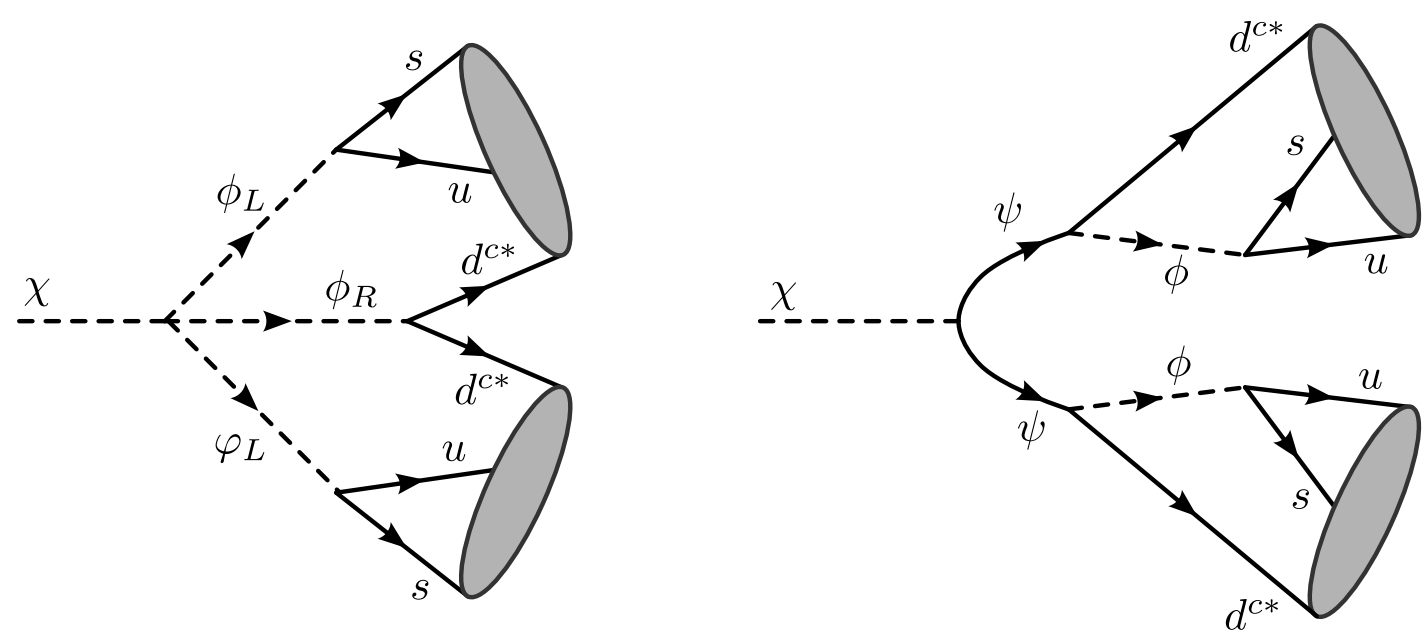

Figure 4. The $\chi$ decay in the MFV mediator model through the off-shell scalar mediators $\phi_{L, R}, \varphi_{L}$ (left), and through the off-shell fermion $\psi$ and scalar $\phi$ mediators in the FN model (right).

baryon number conserving interaction Lagrangian are

$$
\mathcal{L}_{\mathrm{INT}} \supset \frac{g_{q, A B}}{2} \phi_{\gamma}\left(q_{A, \alpha i}^{* j} q_{B, \beta j}^{* k}\right) \epsilon^{i j} \epsilon^{\alpha \beta \gamma}+g_{d, A} \phi^{* \alpha}\left(d_{A, \alpha}^{c} \psi\right)+\frac{g_{\chi}}{2} \chi\left(\psi^{c} \psi^{c}\right)+\text { h.c. },
$$

where, for the couplings $g_{q}, g_{d}$, we also denote the flavor dependence. If the flavor breaking is of the FN type and the mediators do not carry a horizontal charge, then

$$
g_{q, A B} \sim g_{q} \lambda^{\left|H\left(q_{A}\right)+H\left(q_{B}\right)\right|}, \quad g_{d, A} \sim g_{d} \lambda^{\left|H\left(d_{A}\right)\right|},
$$

where $g_{q, d} \sim \mathcal{O}(1)$.

Integrating out the mediators generates the operator (3.13) with the Wilson coefficient

$$
\begin{aligned}
\frac{\mathcal{C}}{\Lambda^{6}} & \simeq \frac{1}{8 m_{\psi}^{2} m_{\phi}^{4}} g_{\chi} g_{q, M, K^{\prime}} g_{q, N^{\prime} M^{\prime}} g_{d, K} g_{d, N} \\
& \sim \frac{1}{8 m_{\psi}^{2} m_{\phi}^{4}} \lambda^{\left|H\left(d_{K}^{c}\right)\right|+\left|H\left(d_{N}^{c}\right)\right|+\left|H\left(q_{M}\right)+H\left(q_{K^{\prime}}\right)\right|+\left|H\left(q_{N}^{\prime}\right)+H\left(q_{M^{\prime}}\right)\right|} .
\end{aligned}
$$

Note that the flavor suppression here is parametrically different than in (3.14) which was obtained by assuming that the FN scale is close to the TeV scale and that the interactions of DM with the visible sector involve the FN fields. In the above model, however, the FN scale can be arbitrarily high and only fixes the flavor interactions between the mediator and the SM fields. Consequently, the leading decay is now $\chi \rightarrow$ ussuds where the suppression for the amplitude is $\sim \lambda^{\left|H\left(d^{c}\right)\right|+\left|H\left(s^{c}\right)\right|+2\left|H\left(q_{2}\right)+H\left(q_{1}\right)\right|} \sim \lambda^{15}$, to be compared with the $\lambda^{4}$ suppression in the more conservative case considered in section 3.2 where the leading decay is $\chi \rightarrow u d s u d s$. The indirect detection bound (4.2) thus translates in our toy mediator model to $m_{\phi, \psi} \gtrsim 130 \mathrm{GeV}$ for mass degenerate $\phi$ and $\psi$. However, since the coupling to the third generation quarks is $\mathcal{O}(1)$, the scalar mediators should in fact be heavier than the top quark in order not to modify its total decay width.

The scaling (5.4) changes if the mediators carry nonzero horizontal charges. For instance, if the horizontal charge of $\phi$ is nonzero, $H(\phi) \neq 0$, one has $g_{q, A B} \sim$ 

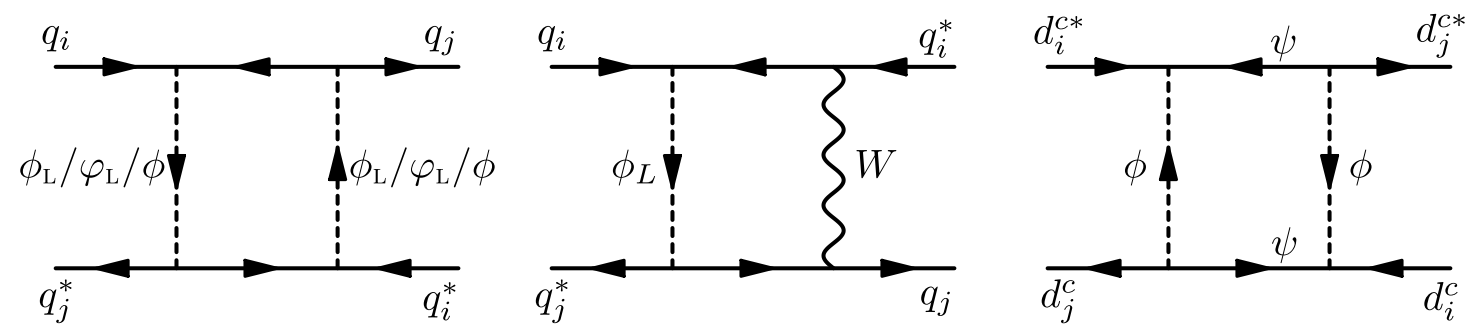

Figure 5. Box diagrams contributing to the neutral meson mixing. In the MFV model, there is also a contribution with both $\phi_{L}$ and $\varphi_{L}$ in the loop, while $\phi_{R}$ contributions are suppressed and can be ignored.

$\lambda^{\left|H\left(q_{A}\right)+H\left(q_{B}\right)-H(\phi)\right|}, g_{d, A} \sim \lambda^{\left|H\left(d_{A}\right)+H(\phi)\right|}$. In this case, the indirect detection bounds need to be appropriately rescaled. For $-2 \leq H(\phi) \leq 5$ the Wilson coefficient is still given by (5.5) and thus $m_{\phi, \psi} \gtrsim 130 \mathrm{GeV}$ from indirect bounds as before. For other values of $H(\phi)$, the bound becomes even weaker.

As far as the annihilation of symmetric part of the $\chi$ relic density is concerned, similar comments as for the MFV model in section 5.1 apply. The dark U(1) is phenomenologically not viable, while annihilation to light scalars is. Furthermore, if $\psi$ has a mass within $\mathcal{O}(10 \%)$ of $m_{\chi}$, the process $\chi \chi^{\dagger} \rightarrow \psi \psi^{\dagger}$, which is forbidden at zero temperature but allowed for nonzero temperatures at the freeze-out, can efficiently annihilate away the symmetric component of $\chi$ without any need for additional states.

\section{Experimental signatures of the mediators}

Now we turn to the experimental signatures of weak scale mediators, the flavor constraints, direct DM detection, and DM production at the LHC.

\subsection{Flavor constraints}

The two mediator models from section 5 do not lead to tree level flavor changing neutral currents (FCNCs). These are first generated at 1-loop, see figure 5. For real couplings $\kappa_{i}$ and $g_{q / d}$ in eqs. (5.1) and (5.3), the constraints from $K^{0}-\bar{K}^{0}, D^{0}-\bar{D}^{0}$ and $B_{(s)}^{0}-\bar{B}_{(s)}^{0}$ mixing require the mediators masses to be generically above several hundred $\mathrm{GeV}$ as we show below. For related analyses of flavor constraints on diquarks, see, e.g., [78, 79].

The $\Delta F=2$ effective weak Hamiltonian is

$$
\mathcal{H}_{\text {eff }}^{\Delta F=2}=\sum_{i} \mathcal{C}_{i} \mathcal{O}_{i}+\sum_{i} \tilde{\mathcal{C}}_{i} \tilde{\mathcal{O}}_{i}
$$

where $i=1, \ldots, 5$ runs over the dimension six operators (we use the notation in [80]). Integrating out the mediators and the $W$ at the weak scale gives, at leading order, a nonzero Wilson coefficient for the operator

$$
\mathcal{O}_{1}=\left(\bar{S}^{\alpha} \gamma_{\mu} P_{L} D^{\alpha}\right)\left(\bar{S}^{\beta} \gamma^{\mu} P_{L} D^{\beta}\right)=\left(s^{* \alpha} \bar{\sigma}^{\mu} d^{\alpha}\right)\left(s^{* \beta} \bar{\sigma}_{\mu} d^{\beta}\right),
$$


in the case of the MFV model, and for both $\mathcal{O}_{1}$ and its parity conjugate operator

$$
\tilde{\mathcal{O}}_{1}=\left(\bar{S}^{\alpha} \gamma_{\mu} P_{R} D^{\alpha}\right)\left(\bar{S}^{\beta} \gamma^{\mu} P_{R} D^{\beta}\right)=\left(s^{c \alpha} \bar{\sigma}^{\mu} d^{c \alpha *}\right)\left(s^{c \beta} \bar{\sigma}_{\mu} d^{c \beta *}\right),
$$

in the case of the FN model. Above, we first give the operators in the 4-component notation and then also in the 2-component notation (for our notation see appendix A).

In the matching there are two types of contributions: in the first, only the mediators run in the loop whereas in the second, both the scalar mediator and the $W$ boson run in the loop, see figure 5. For the MFV model, these give for the $K^{0}-\bar{K}^{0}, D^{0}-\bar{D}^{0}$, and $B_{(s)}-\bar{B}_{(s)}$ mixing

$$
\begin{aligned}
\mathcal{C}_{1 K}^{\mathrm{MFV}}= & \frac{1}{64 \pi^{2} m_{\phi}^{2}}\left\{\left(V_{c s} V_{c d}^{*}\right)^{2}\left[\left(\kappa_{1}^{4}+3 \kappa_{2}^{4}-2 \kappa_{1}^{2} \kappa_{2}^{2}\right) F\left(x_{c}\right)+4 g_{w}^{2} \kappa_{2}^{2} G\left(x_{w}, x_{c}\right)\right]+c \rightarrow t\right. \\
& \left.-2 V_{c s} V_{c d}^{*} V_{t s} V_{t d}^{*}\left[\left(\kappa_{1}^{4}+3 \kappa_{2}^{4}-2 \kappa_{1}^{2} \kappa_{2}^{2}\right) F^{F}\left(x_{c}, x_{t}\right)+4 g_{w}^{2} \kappa_{2}^{2} G^{F}\left(x_{w}, x_{c}, x_{t}\right)\right]\right\}, \\
\mathcal{C}_{1 D}^{\mathrm{MFV}}= & \frac{1}{64 \pi^{2} m_{\phi}^{2}}\left\{\left(V_{u s} V_{c s}^{*}\right)^{2}\left[\left(\kappa_{1}^{4}+3 \kappa_{2}^{4}-2 \kappa_{1}^{2} \kappa_{2}^{2}\right) F\left(x_{s}\right)+4 g_{w}^{2} \kappa_{2}^{2} G\left(x_{w}, x_{s}\right)\right]+s \rightarrow b\right. \\
& \left.-2 V_{u s} V_{c s}^{*} V_{u b} V_{c b}^{*}\left[\left(\kappa_{1}^{4}+3 \kappa_{2}^{4}-2 \kappa_{1}^{2} \kappa_{2}^{2}\right) F^{F}\left(x_{s}, x_{b}\right)+4 g_{w}^{2} \kappa_{2}^{2} G^{F}\left(x_{w}, x_{s}, x_{b}\right)\right]\right\}, \\
\mathcal{C}_{1 B_{q}}^{\mathrm{MFV}=} & \frac{1}{64 \pi^{2} m_{\phi}^{2}}\left(V_{t b} V_{t q}^{*}\right)^{2}\left[\left(\kappa_{1}^{4}+3 \kappa_{2}^{4}-2 \kappa_{1}^{2} \kappa_{2}^{2}\right) F\left(x_{t}\right)+4 g_{w}^{2} \kappa_{2}^{2} G\left(x_{w}, x_{t}\right)\right],
\end{aligned}
$$

where $q=d, s, x_{i}=\left(m_{i} / m_{\phi}\right)^{2}$, and we have set $m_{u}=m_{d}=0$ and assumed, for simplicity, that the $\phi$ and $\varphi$ are mass degenerate. The loop functions $F(x), F^{F}\left(x_{1}, x_{2}\right), G\left(x_{1}, x_{2}\right)$, and $G^{F}\left(x_{1}, x_{2}, x_{3}\right)$ are given in appendix D. As in the SM, the largest contribution to the $K^{0}-\bar{K}^{0}$ mixing is due to the charm-charm loop while, for $B_{q}^{0}-\bar{B}_{q}{ }^{0}$ mixing, the top loop dominates as expected.

For the FN model, the Wilson coefficients are given by

$$
\begin{aligned}
\mathcal{C}_{1 K}^{\mathrm{FN}} \sim \frac{\lambda^{10} g_{q}^{4}}{16 \pi^{2} m_{\phi}^{2}}\left[H\left(x_{t}\right)+2 \lambda^{4} H^{F}\left(x_{c}, x_{t}\right)\right], & \tilde{\mathcal{C}}_{1 K}^{\mathrm{FN}} \sim \frac{\lambda^{10} g_{d}^{4}}{16 \pi^{2} m_{\phi}^{2}} H\left(x_{\psi}\right), \\
\mathcal{C}_{1 B_{d}}^{\mathrm{FN}} \sim \frac{\lambda^{6} g_{q}^{4}}{16 \pi^{2} m_{\phi}^{2}}\left[H\left(x_{t}\right)+2 \lambda^{4} H^{F}\left(x_{c}, x_{t}\right)\right], & \tilde{\mathcal{C}}_{1 B_{d}}^{\mathrm{FN}} \sim \frac{\lambda^{10} g_{d}^{4}}{16 \pi^{2} m_{\phi}^{2}} H\left(x_{\psi}\right), \\
\mathcal{C}_{1 B_{s}}^{\mathrm{FN}} \sim \frac{\lambda^{4} g_{q}^{4}}{16 \pi^{2} m_{\phi}^{2}}\left[H\left(x_{t}\right)+2 \lambda^{4} H^{F}\left(x_{c}, x_{t}\right)\right], & \tilde{\mathcal{C}}_{1 B_{d}}^{\mathrm{FN}} \sim \frac{\lambda^{8} g_{d}^{4}}{16 \pi^{2} m_{\phi}^{2}} H\left(x_{\psi}\right),
\end{aligned}
$$

while $\mathcal{C}_{1 D}^{\mathrm{FN}}=\mathcal{C}_{1 K}^{\mathrm{FN}}$ and $\tilde{\mathcal{C}}_{1 D}^{\mathrm{FN}}=0$. Above, we have indicated the scaling of different contributions to the Wilson coefficient in terms of $\lambda=0.2$, cf. section 3.2. In the numerics we use the equality sign. The loop functions $H(x)$ and $H^{F}\left(x_{1}, x_{2}\right)$ are given in appendix D.

Note that the above Wilson coefficients contain $\log \left(m_{i} / m_{\phi}\right)$ that can become large for $m_{\phi} \gg m_{i}$. We do not attempt to resum these logarithms which also means that we treat all the NP contributions as local. We expect that our numerical results can receive $\mathcal{O}(1)$ corrections due to neglected terms. This is within the precision required for our analysis. 


\begin{tabular}{|ccccc|}
\hline & \multicolumn{2}{c}{ MFV } & \multicolumn{2}{c|}{ FN } \\
& $\kappa_{1,2}<$ & $m_{\phi_{L}, \varphi_{L}}>$ & $g_{q, d}<$ & $m_{\phi}>$ \\
\hline$K^{0}-\bar{K}^{0}$ & 0.33 & $2.9 \mathrm{TeV}$ & 0.63 & $570 \mathrm{GeV}$ \\
$B_{d}-\bar{B}_{d}$ & 1.3 & $710 \mathrm{GeV}$ & 0.54 & $1 \mathrm{TeV}$ \\
$B_{s}-\bar{B}_{s}$ & 1.3 & $780 \mathrm{GeV}$ & 0.59 & $840 \mathrm{GeV}$ \\
$D^{0}-\bar{D}^{0}$ & 30 & $34 \mathrm{GeV}$ & 4.3 & $56 \mathrm{GeV}$ \\
\hline
\end{tabular}

Table 4. The $95 \%$ C.L. bounds on the MFV and FN mediator models from meson mixing. Taking $m_{\phi_{L}}=m_{\varphi_{L}}=m_{\phi}=1 \mathrm{TeV}$ and $\kappa_{1}=\kappa_{2}\left(g_{q}=g_{d}\right)$ gives the upper bounds on the couplings in the 2nd(4th) column. Taking in turn $\kappa_{1,2}=g_{q, d}=1$ gives lower bounds on the mediator masses in the $3 \mathrm{rd}$ and 5 th columns. The mass of the fermion in the FN model is fixed to $m_{\psi}=20 \mathrm{GeV}$ (see section 6.3). The bounds are not very sensitive to $m_{\psi}$.
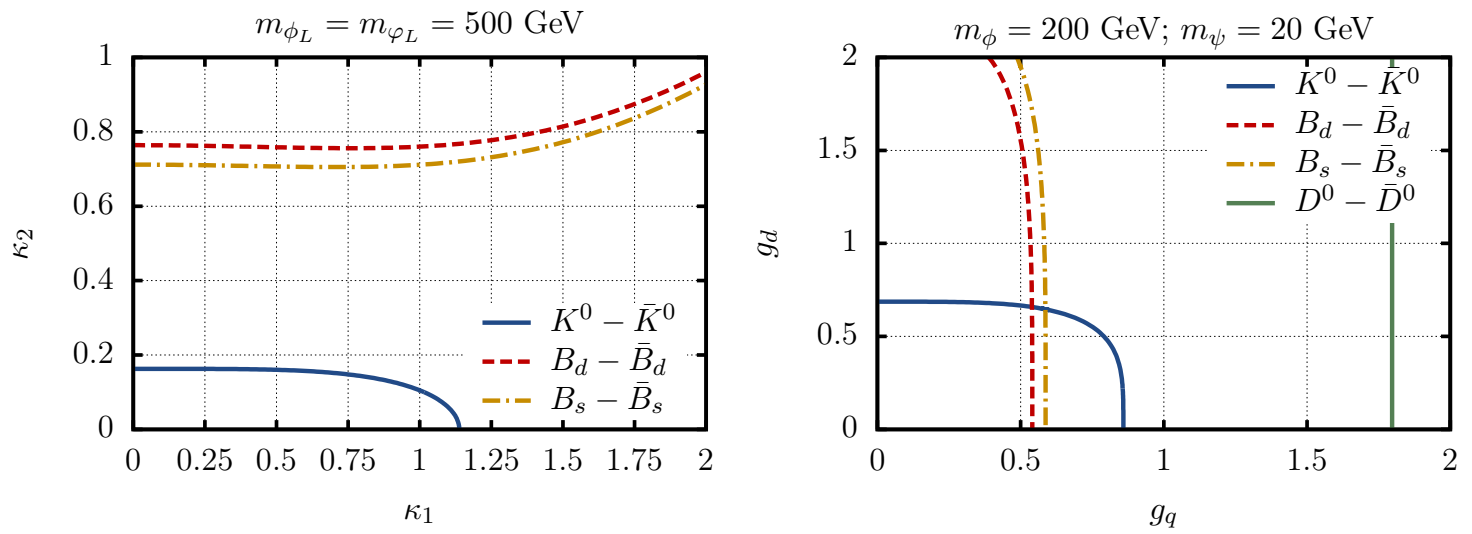

Figure 6. Meson mixing constraints on the couplings $\kappa_{1,2}$ in the MFV mediator model (left) and $g_{q, d}$ in the FN model (right), taking $m_{\phi_{L}}=m_{\varphi_{L}}=500 \mathrm{GeV}$ and $m_{\phi}=200 \mathrm{GeV}, m_{\psi}=20 \mathrm{GeV}$ respectively. The excluded regions lie above and to the right of the curves.

Though, we do include the usual RGE effects due to the NLO QCD running of the effective weak Hamiltonian from the weak scale to the low energy. For constraints from $K^{0}-\bar{K}^{0}$ and $B_{(s)}-\bar{B}_{(s)}$ mixing we use the recent results of a fit to the mixing parameters in [81]. The constraints from $D^{0}-\bar{D}^{0}$ mixing are obtained by assuming that the NP contribution saturates $\Delta m_{D}$ so that in the equation $x_{D}=2\left|\left\langle\bar{D}^{0}\left|\mathcal{H}_{\text {eff }}^{\Delta C=2}\right| D^{0}\right\rangle\right| / \Gamma_{D}$, valid in the limit of no CP violation, we only include the NP contribution [80]. The resulting bounds on couplings and masses are shown in table 4 . In the case of the MFV model, the most severe bound comes from $K^{0}-\bar{K}^{0}$ and is due to $\epsilon_{K}$. Since we assume that all the $\kappa_{i}$ in (5.1) are real, the NP contribution does carry a weak phase due to the $V_{t s} V_{t d}^{*}$ CKM factors and does contribute to $\epsilon_{K}$. In contrast, in the FN model the NP contributions to the mixing do not carry a weak phase and thus do not have an effect on $\epsilon_{K}$. Therefore, the bounds from $K^{0}-\bar{K}^{0}$ mixing are much less severe.

In figure 6 , we show the constraint on the couplings $\kappa_{1,2}$ in the MFV model, fixing $m_{\phi_{L}}=m_{\varphi_{L}}=500 \mathrm{GeV}$ (left figure), and the constraints on $g_{q, d}$ in the FN model, fixing $m_{\phi}=200 \mathrm{GeV}, m_{\psi}=20 \mathrm{GeV}$ (right figure). Since, in the case of MFV, the largest 
contribution to $K^{0}-\bar{K}^{0}$ is from the mediator- $W$ loop, the $\epsilon_{K}$ bound places a stringent constraint on $\kappa_{2}$. Since the NP contributions to the meson mixing were assumed to be CP conserving in the case of the FN model, the couplings $g_{d, q} \sim \mathcal{O}(1)$ are allowed even for $m_{\phi}$ as low as $200 \mathrm{GeV}$.

\subsection{Relic abundance and direct detection}

We note in passing that the virtual exchanges of the mediators generate contact operators of the schematic form $\chi^{\dagger} \chi \bar{q} q$ that contribute to the $\chi \chi^{\dagger}$ annihilation cross section and to the cross section for DM scattering on nuclei. The symmetric couplings of DM and the mediators, of schematic form $\chi \chi^{\dagger} \phi \phi^{\dagger}$, do not suffice to create large enough annihilation cross sections that would annihilate away the symmetric component of DM relic abundance.

As an example, consider the MFV model with scalar mediators, eq. (5.1), and assume that the lightest mediator is $\phi_{L}$. It can have a symmetric coupling to DM of the form

$$
\mathcal{L} \supset \kappa^{\prime}\left[\phi_{L}\right]_{\gamma}^{I}\left[\phi_{L}^{\dagger}\right]_{\gamma}^{I} \chi^{\dagger} \chi
$$

At 1-loop, this generates a contact interaction $\chi^{\dagger} \partial_{\mu} \chi \bar{q} \gamma^{\mu} q$, which leads to an annihilation cross section $\langle\sigma v\rangle \sim \mathcal{O}\left(10^{-28} \mathrm{~cm}^{3} / \mathrm{s}\right)\left(100 \mathrm{GeV} / m_{\phi_{L}}\right)^{4}$ for $\mathcal{O}(1)$ couplings. This annihilation cross section is more than three orders of magnitude too small to obtain the observed relic density and satisfy CMB constraints for $s$-wave annihilation [57]. Thus, the symmetric component of the DM needs to annihilate away through a different mechanism as discussed at the end of section 5.1.

\subsection{Collider signatures}

In both the MFV and FN flavor breaking scenarios, the mediator models involve colored scalars. These can be searched for at the LHC through the gluon initiated pair production or through a single production. We use our two mediator models to estimate the LHC reach. The MFV mediator model, eq. (5.1), contains three colored scalars that are either triplets or sextets of the color and flavor groups, see table 2. The FN model, eq. (5.3), contains a colored scalar and a neutral fermion, see table 3 .

Pair production of colored scalars is the dominant production mechanism of the mediators for the masses of interest, below $\mathcal{O}(\mathrm{TeV})$. We illustrate this in figure 7 for the color triplet $\phi$ in the FN model where we compare the pair production cross section from gluon fusion and from quark-guon fusion, and the single production of $\phi$ in association with a jet. Gluon fusion clearly dominates in the mass range of interest.

The signatures of pair produced colored scalars depend on their decay modes. In our two models they decay either directly to two SM quarks or, alternatively, first to two lighter scalars that then in turn decay to two jets each. In the FN model the decay $\phi \rightarrow j \psi$ is also possible. The flavor composition of the jets depends on the flavor quantum numbers of the scalar. For instance, the states in the $\phi_{L}$ flavor multiplet can decay either predominantly through $\phi_{L} \rightarrow t b, \phi_{L} \rightarrow b j$, or $\phi_{L} \rightarrow j j$, depending on the flavor numbers of $\phi_{L}$ (and similarly for $\varphi_{L}$ ), see eq. (5.1). The scalars in the $\phi_{R}$ flavor multiplet, on the other hand, decay through $\phi_{R} \rightarrow b j$ or $\phi_{R} \rightarrow j j$, again depending on the flavor index carried by the 


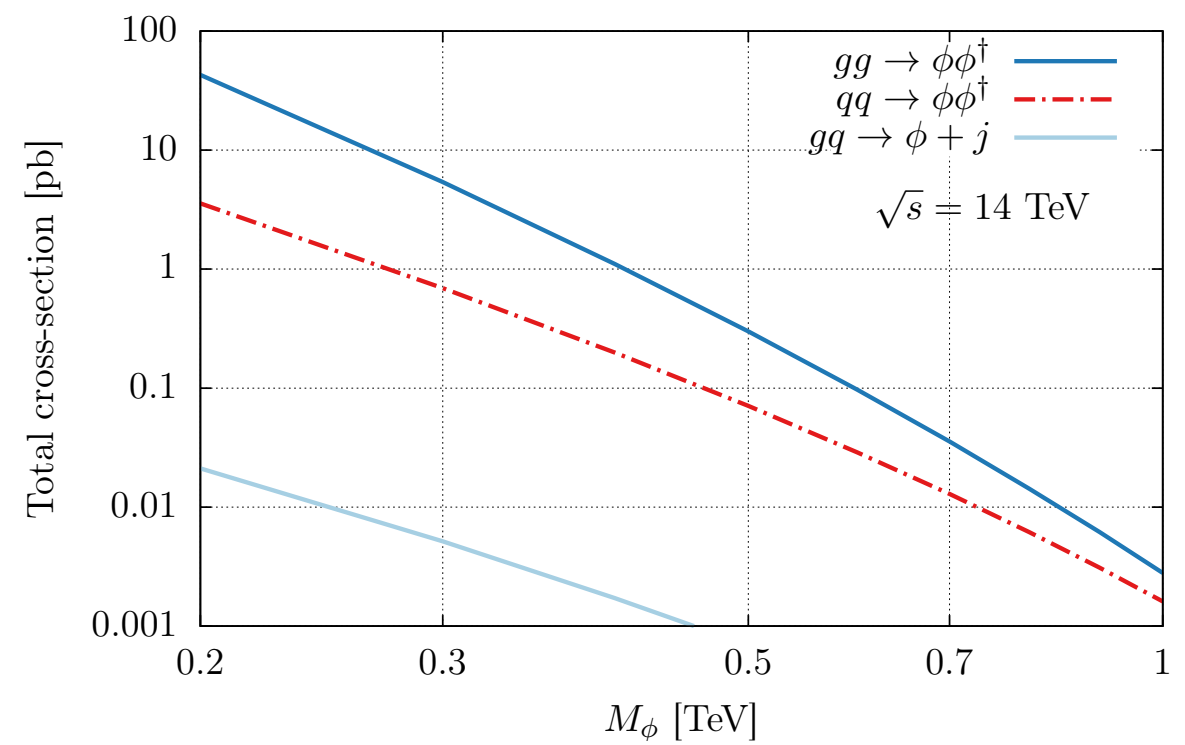

Figure 7. The $g g \rightarrow \phi \phi^{\dagger}$ (solid blue), $q q \rightarrow \phi \phi^{\dagger}$ (dot-dashed red) and $g q \rightarrow \phi j$ (solid light blue) contributions to the pair-production and single-production cross-section at the LHC with $\sqrt{s}=14 \mathrm{TeV}$ as a function of a mass of a color triplet scalar $\phi$, a mediator in the FN model.

$\phi_{R}$ state. In the FN model one needs to require $m_{\phi}>m_{t}$ in order not to modify the total decay width of the top quark, see section 5 . Then, the dominant decay is either $\phi \rightarrow \bar{b} \psi$ or $\phi \rightarrow t b$, depending on the relative sizes of the two couplings, while the other decays are suppressed by additional powers of $\lambda$.

To get a rough estimate of the LHC sensitivity we treat all the decay modes as twojet final states (this overestimates the reach slightly since, for the $t j$ final state, the real efficiency is expected to be lower). The strongest constraint on pair-production of the lightest scalar mediators then comes from the search for pair-produced dijet resonances from CMS at $7 \mathrm{TeV}$ LHC with integrated luminosity of $5 \mathrm{fb}^{-1}$ [82]. This places the bounds $m_{\phi} \gtrsim 470 \mathrm{GeV}$ in the case of FN model assuming that $\phi \rightarrow \bar{b} \psi$ decay is negligible, and $m_{\phi_{L}} \gtrsim 620 \mathrm{GeV}, m_{\varphi_{L}} \gtrsim 910 \mathrm{GeV}, m_{\phi_{R}} \gtrsim 580 \mathrm{GeV}$ in the case of MFV flavor breaking as shown in figure 8. Note that when all three mediators are degenerate in mass, the color sextet scalar has the largest pair production cross section due to the large color factor.

In the FN model, a new experimental signature is obtained in the limit $g_{d} \gg \lambda^{2} g_{q}$. Then the dominant decay of $\phi$ is $\phi \rightarrow \bar{b} \psi$. In order not to have fast decaying DM $m_{\psi}>$ $m_{\chi} / 2$. Using NDA the $\psi$ decay length is

$$
c \tau(\psi \rightarrow b b c) \sim\left(g_{q}^{2} g_{d}^{2} \lambda^{8} \frac{1}{8 \pi} \frac{1}{16 \pi^{2}} \frac{m_{\psi}^{5}}{m_{\phi}^{4}}\right)^{-1} \sim 30 \mathrm{~m}\left(\frac{20 \mathrm{GeV}}{m_{\psi}}\right)^{5}\left(\frac{m_{\phi}}{750 \mathrm{GeV}}\right)^{4}\left(\frac{0.03}{g_{q} g_{d}}\right)^{2} .
$$

For light enough $\psi$ (or heavy enough $\phi$ ), the fermion $\psi$ does not decay in the detector and appears as $\mathbb{F}_{T}$. The $p p \rightarrow \phi \phi^{\dagger}$ pair production then results in $2 j+\mathbb{E}_{T}$ or $2 b+\mathbb{F}_{T}$ final state, and is bounded from sbottom searches as shown in figure 9. The resulting bound is $m_{\phi}>550(760) \mathrm{GeV}$ for $m_{\psi}=20 \mathrm{GeV}$ and $\mathcal{B R}(\phi \rightarrow \psi b)=0.5(1.0)$. The choice 


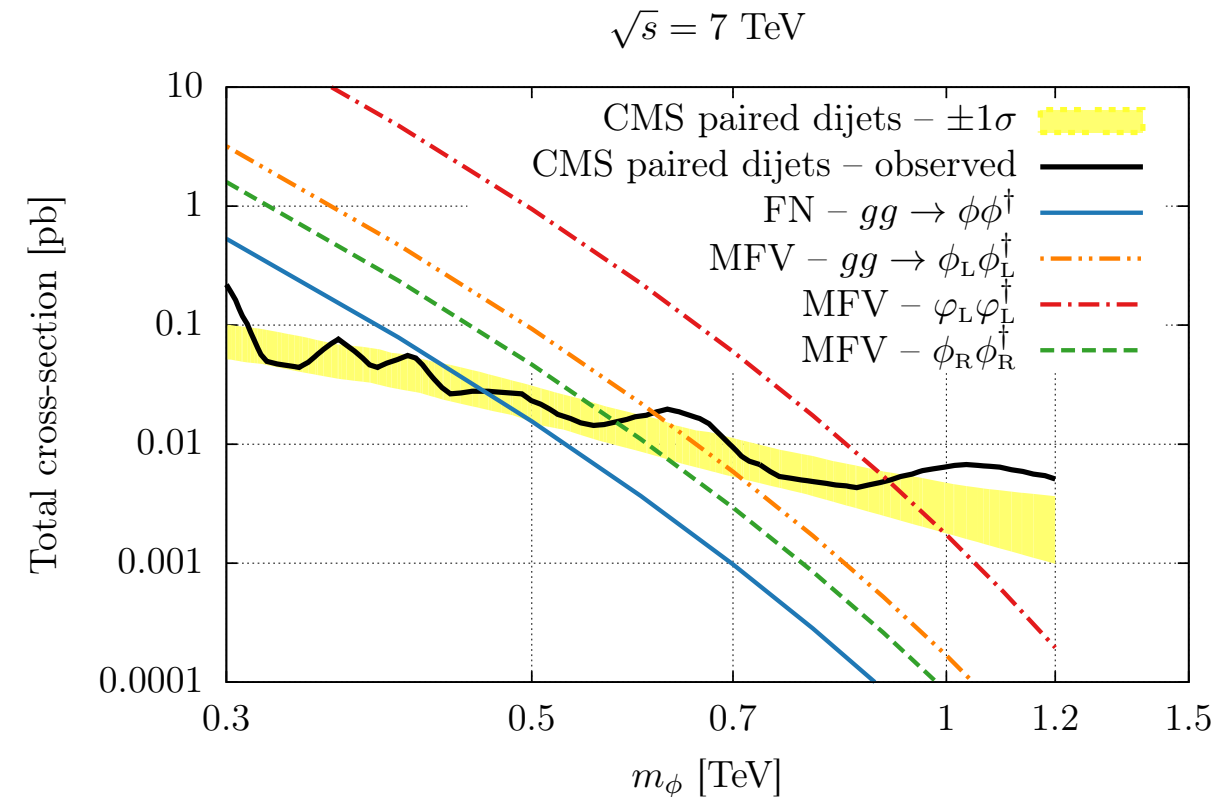

Figure 8. Constraints on the scalar mediator $\phi$ in the $\mathrm{FN}$ model, and $\phi_{L}, \varphi_{L}, \phi_{R}$ in the MFV model that follow from the CMS search for pair-produced dijet-resonances [82]. The states in the same flavor multiplet are taken to be mass-degenerate.

$g_{q}=g_{d}=0.03$ in eq. (6.11) gives $\mathcal{B R}(\phi \rightarrow b \psi) \approx \mathcal{B} \mathcal{R}(\phi \rightarrow s \psi)=0.33$. For the same input parameters, the single production of $\psi$ in association with $b, t$, or $\phi$ has a cross section $\sim 7 \cdot 10^{-2} \mathrm{fb}$ while the pair production is dominated by the process $s s \rightarrow \psi \psi$ and has a negligible cross section of $\sim 4 \cdot 10^{-4} \mathrm{fb}$.

The single production of mediators, e.g., $u d \rightarrow \phi, u d \rightarrow \phi_{L}, u d \rightarrow \varphi_{L}, d s \rightarrow \phi_{R}$, is suppressed due to the small couplings of the mediators to the first and the second generation quarks. Similarly, the single production from heavy quarks in the initial state suffers from the PDF suppression.

For single top production, the MFV model gives the largest contribution with a crosssection of $\sigma\left(u d \rightarrow \phi_{L} \rightarrow t b\right)_{\mathrm{MFV}}=7.6 \times 10^{-6} \mathrm{pb}$ for $m_{\phi_{L}}=500 \mathrm{GeV}$ and $\sqrt{s}=8 \mathrm{TeV}$. This is well below the SM production cross section. Thus, the ATLAS and CMS combined measurement of the single top cross section at $\sqrt{s}=8 \mathrm{TeV}, 85 \pm 12 \mathrm{pb}$ [84] and so does not impose any limits on the mediator model.

The production of the DM, $\chi$, can occur from the decay of heavier mediators. For instance, for $\kappa_{4} \sim \kappa_{3}$ and $\phi_{R}$ heavy enough, the dominant decay mode of $\phi_{3}$ is $\phi_{3} \rightarrow \chi \varphi_{L}^{\dagger} \phi_{L}^{\dagger}$. Pair production $p p \rightarrow \phi_{R} \phi_{R}^{\dagger}$ would thus result in $8 j+\mathbb{F}_{T}$ signature where paired dijets would reconstruct $\phi_{L}$ and $\varphi_{L}$ mass peaks (depending on the flavor assignments some of the jets can be replaced by $t$ of $b$ jets).

\section{Conclusions}

We showed that for asymmetric DM (ADM) models, the stability of DM on cosmological time scales may be purely accidental. We do not require that the DM to be charged under 


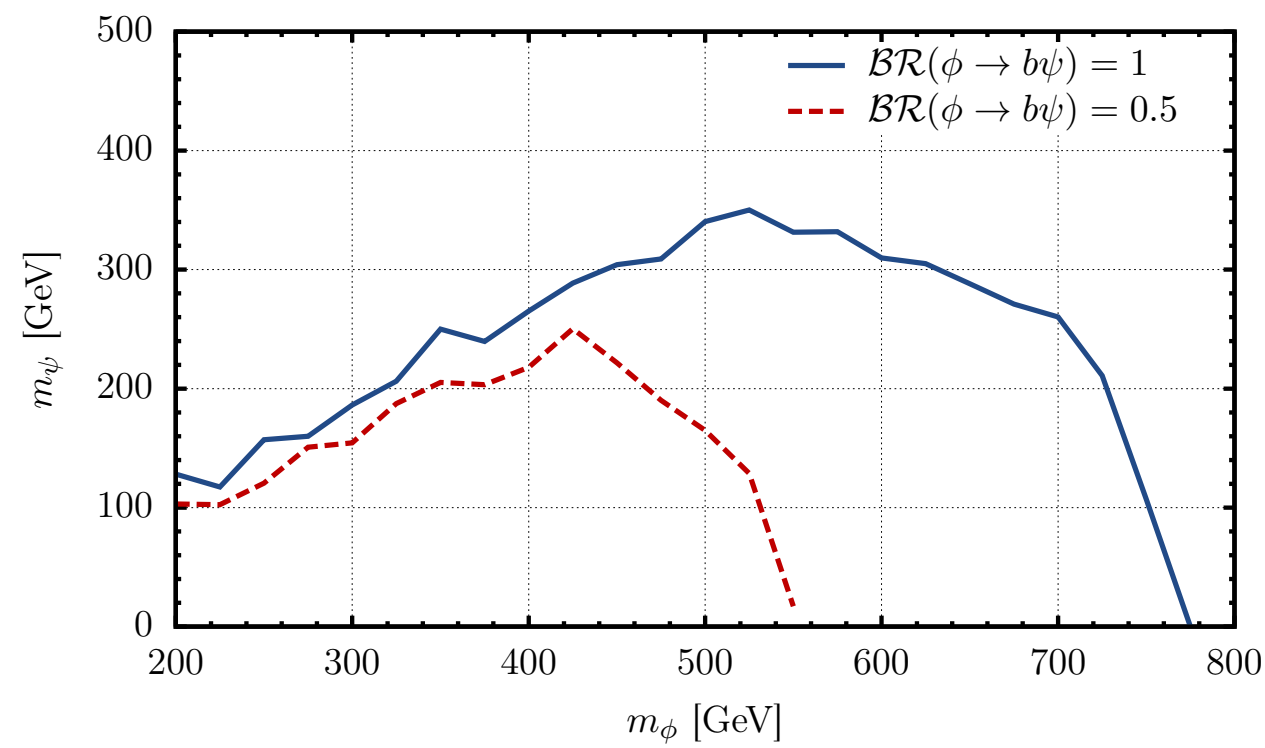

Figure 9. The $95 \%$ exclusion limit on $\phi \phi^{\dagger}$ production in the FN model for the $b \bar{b} \psi \bar{\psi}$ final state, where $\psi$ escapes the detector and sbottom search applies [83]. The solid blue (dashed red) line is for $\phi \rightarrow b \psi$ branching ratios of $50 \%$ and $100 \%$.

an ad-hoc conserved $Z_{n}$ symmetry. Rather, we assume that such a discrete symmetry is explicitly broken by the mediator interactions that transfer the $B-L$ between the DM sector and the visible sector in the early universe. Such asymmetric interactions are necessary in all models of ADM though they may be made to obey a $Z_{4}$ symmetry (i.e. one can demand that they involve only the $\chi \chi \rightarrow$ visible or $\chi^{\dagger} \chi^{\dagger} \rightarrow$ visible transitions instead of $\chi \rightarrow$ visible transitions as in our case).

At low energies, the DM then carries a conserved $\chi$ charge that is broken only by the higher dimensional operators obtained by integrating out the mediators. Such operators also lead to DM decays. In this paper we explored the role of continuous flavor symmetries for the properties of such decaying DM focusing on the case where DM that carries nonzero baryon number. For $B=1 \mathrm{DM}$, the direct detection bounds are evaded if the mediators are above $\sim 4 \cdot 10^{9} \mathrm{TeV}$ assuming $\mathcal{O}(1)$ couplings. However, if quark flavor breaking is of the MFV type, the mediators can be lighter by around two orders of magnitude. For $B=2 \mathrm{DM}$, the scale of the mediators can be much lighter $(\mathcal{O}(8 \mathrm{TeV})$ for $\mathcal{O}(1)$ couplings). This is then lowered by an order of magnitude if quark flavor breaking is of the MFV or Froggatt-Nielsen type. The mediators that would lead to indirect DM signals in the next generation of experiments can thus be, at the same time, searched for at the LHC.

We have explored this possibility by constructing two mediator models, one with assumed MFV and one with a FN flavor breaking pattern. The MFV mediator model (eq. (5.1)) contains three colored scalars that are either triplets or sextets of the color and the flavor groups, see table 2. The FN model (eq. (5.3)), on the other hand, contains 
one colored scalar and one neutral fermion, see table 3. These mediators generate FCNCs at 1-loop. While this leads to nontrivial constraints on their masses and couplings, the mediators can still be as light as a few $\times 100 \mathrm{GeV}$ with $\mathcal{O}(1)$ couplings. Since the mediators are charged under QCD, they can be singly or pair-produced at the LHC with large cross sections. This means that the searches at the LHC can lead to interesting constraints or discoveries. The signatures depend on how the mediators decay. In the FN model, for instance, the decay to heavy quarks, $\phi \rightarrow t b$, is favored. Modifying the paired dijet searches to the $p p \rightarrow \phi \phi \rightarrow t b \bar{t} \bar{b}$ signal could thus enhance the reach of the LHC in the search for these mediators. In the MFV model, on the other hand, paired light dijets, paired $t b$, and paired $b j$ are possible. Other signatures are discussed in section 6.3.

In conclusion, $\mathrm{ADM}$ can quite generically be metastable with a possibility of complementary signals in indirect detection and at the LHC.

\section{Acknowledgments}

J. Z. and F.B. are supported by the U.S. National Science Foundation under CAREER Grant PHY-1151392. F.B. is supported by the Fermilab Fellowship in Theoretical Physics. Fermilab is operated by Fermi Research Alliance, LLC, under Contract No. DE-AC0207CH11359 with the United States Department of Energy. J.Z. is grateful to the Mainz Institute for Theoretical Physics (MITP) for its hospitality and its partial support during the completion of this work. J.Z. thanks the Aspen Center for Physics, supported by the NSF Grant \#1066293, and the KITP, supported in part by the National Science Foundation under Grant No. NSF PHY11-25915, for their warm hospitality. F.B. thanks Prateek Agrawal, Roni Harnik, and Felix Yu for helpful discussions. Some of the cross-sections were computed using MadGraph5 [85] using a model file generated by FeynRules 2.0 [86].

\section{A Operators in four component notation}

In the paper we are using a two-component notation, where the left-handed Weyl fermion fields $\left(q_{i}, u^{c}, d^{c}, l_{i}, e^{c}\right)$ have hypercharges $(+1 / 6,-2 / 3,+1 / 3,-1 / 2,+1)$ and $B-L$ charges $(1 / 3,-1 / 3,-1 / 3,-1,+1)$. The higgs doublet is denoted by $H$ and has $Y=+1 / 2$, while $\tilde{H}=i \sigma_{2} H^{*}$. The two Weyl spinors of the DM Dirac fermion are $\psi$ and $\psi^{c}$ with $B-L=-1$ and +1 , respectively. Finally, $\phi$ is the complex scalar DM with $B-L=2$. Capital letters denote four-component spinors following the notation in [87]. The DM Dirac fermion $\Psi$ and its charge conjugate $\Psi^{C}$ are

$$
\Psi=\left(\begin{array}{c}
\psi_{\alpha} \\
\psi^{c \dagger \dot{\alpha}}
\end{array}\right), \quad \Psi^{C}=\left(\begin{array}{c}
\psi_{\alpha}^{c} \\
\psi^{\dagger \dot{\alpha}}
\end{array}\right)
$$

while $\bar{\Psi}=\left(\psi^{c \alpha}, \psi_{\dot{\alpha}}\right)$. Writing for the two-component spinors $q_{1}=u_{L}, q_{2}=d_{L}$ and $\ell_{1}=\nu_{L}$, $\ell_{2}=e_{L}$, suppressing generation indices, we introduce

$$
U=\left(\begin{array}{c}
u_{L \alpha} \\
u^{c \dagger \dot{\alpha}}
\end{array}\right), \quad D=\left(\begin{array}{c}
d_{L \alpha} \\
d^{c \dagger \dot{\alpha}}
\end{array}\right), \quad E=\left(\begin{array}{c}
e_{L \alpha} \\
e^{c \dagger \dot{\alpha}}
\end{array}\right), \quad N=\left(\begin{array}{c}
\nu_{L \alpha} \\
\nu^{c \dagger \dot{\alpha}}
\end{array}\right),
$$


where $\nu^{c}$ is the right-handed neutrino field introduced for completeness. If neutrino is Majorana, $\nu_{L}=\nu^{c}$. The weak doublets in the four-component notation are

$$
Q_{L}=\left(U_{L}, D_{L}\right), \quad L_{L}=\left(N_{L}, E_{L}\right),
$$

with $U_{L} \equiv P_{L} U$, etc, and similarly $U_{R} \equiv P_{R} U, D_{R} \equiv P_{R} D, \ldots$. Some examples of the relevant asymmetric operators in the two- and four-component notations are given below.

$$
\begin{array}{ll}
\underline{\operatorname{dim} 6:} & \mathcal{Q}_{1}^{(6)}=\left(q_{i} q^{i}\right)\left(d^{c *} \psi^{c *}\right)=\left(\overline{Q_{L i}^{C}} Q_{L}^{i}\right)\left(\overline{D^{C}} P_{R} \Psi\right), \\
& \mathcal{Q}_{2}^{(6)}=\left(u^{c} d^{c}\right)\left(d^{c} \psi^{c}\right)=\left(\bar{U} P_{L} D^{C}\right)\left(\bar{D} P_{L} \Psi^{C}\right), \\
\underline{\operatorname{dim} 10:} & \mathcal{Q}_{1}^{(10)}=\phi\left(d^{c} d^{c}\right)\left(q_{i}^{*} q^{i *}\right)\left(q_{j}^{*} q^{j *}\right)=\phi\left(\bar{D} P_{L} D^{C}\right)\left(\overline{Q_{L i}} P_{R} Q_{L}^{C i}\right)\left(\overline{Q_{L i}} P_{R} Q_{L}^{C i}\right) .
\end{array}
$$

\section{B Asymmetric DM relic density}

Here we review the relations between the DM relic density and the DM mass in ADM models. We assume that the operator(s) transferring the $B-L$ asymmetry from the visible to the dark sector decouple above electroweak phase transition, $T_{C}>T_{\text {ew }} \sim 170 \mathrm{GeV}$ [88], as is the case for our ADM models, see section 2. We first assume that the visible sector consists below $T_{C}$ of only the SM fields (we will later relax this). The number density asymmetry for relativistic particles is

$$
(n-\bar{n})_{i}=\frac{T^{3}}{6} \hat{g}_{i} \frac{\mu_{i}}{T}
$$

where $n(\bar{n})$ are the particle(anti-particle) number densities, $\mu_{i}$ is the chemical potential for species $i$, and $\hat{g}_{i}=g_{i}\left(g_{i} / 2\right)$ for bosons (fermions) with $g_{i}$ internal degrees of freedom so that $\hat{g}_{i}=1$ for a Weyl fermion, while $\hat{g}_{i}=2$ for a Dirac fermion or a complex scalar.

All the SM particles are in chemical equilibrium, so that the chemical potentials are proportional to the conserved quantum numbers [89]. Above the electroweak phase transition these are $B-L, Y$ and $\mathrm{SU}(2)_{L}$, while $B+L$ is broken by sphalerons. Thus (see also [90])

$$
\mu_{i}=\left(T_{3}\right)_{i} c_{3}+Y_{i} c_{Y}+(B-L)_{i} c_{B-L}
$$

where the $c_{i}$ are constants that we determine from net weak isospin, hypercharge and $B-L$ densities. The net weak isospin charge density in the universe normalized to entropy density is

$$
T_{3} \propto \sum_{i} \hat{g}_{i}\left(T_{3}\right)_{i} \mu_{i}=\sum_{i} \hat{g}_{i}\left(T_{3}\right)_{i}^{2} c_{3}+0 \cdot c_{Y}+0 \cdot c_{B-L}=0 .
$$

For the first equality we used that for each $\mathrm{SU}(2)$ multiplet $\sum_{i}\left(T_{3}\right)_{i}=0$, and in the second equality that the net $T_{3}$ charge is zero since $\mathrm{SU}(2)_{L}$ is not explicitly broken. Thus $c_{3}=0$ and the $\mathrm{SU}(2)_{L}$ charge of a particle does not contribute to its chemical potential.

Flavor mixing ensures that the chemical potentials for SM Weyl fermions from different generations are the same. Similarly, $\mathrm{SU}(2)_{L}$ interactions ensure that $\mu_{u_{L}}=\mu_{d_{L}} \equiv \mu_{Q}$, and $\mu_{\ell_{L}}=\mu_{\nu} \equiv \mu_{L}$. We thus have

$$
\begin{gathered}
\mu_{H}=\frac{1}{2} c_{Y}, \quad \mu_{L}=-\frac{1}{2} c_{Y}-c_{B-L}, \quad \mu_{E}=-c_{Y}-c_{B-L}, \\
\mu_{Q}=\frac{1}{6} c_{Y}+\frac{1}{3} c_{B-L}, \mu_{U}=\frac{2}{3} c_{Y}+\frac{1}{3} c_{B-L}, \mu_{D}=-\frac{1}{3} c_{Y}+\frac{1}{3} c_{B-L},
\end{gathered}
$$


while for the gauge bosons $\mu_{G}=\mu_{W}=\mu_{B}=0$. The net hypercharge of the universe is thus

$$
\begin{aligned}
Y & \propto \sum_{i} \hat{g}_{i}(Y)_{i} \mu_{i}=\frac{1}{2} 2 \cdot 2 \mu_{H}+N_{f}\left[-\frac{1}{2} 2 \mu_{L}-\mu_{E}+N_{c}\left(\frac{1}{6} \cdot 2 \mu_{Q}+\frac{2}{3} \mu_{U}-\frac{1}{3} \mu_{D}\right)\right] \\
& =2 \mu_{H}+N_{f}\left(\mu_{Q}+2 \mu_{U}-\mu_{D}-\mu_{L}-\mu_{E}\right)=11 c_{Y}+8 c_{B-L},
\end{aligned}
$$

where $N_{f}=3$ is the number of generations and $N_{c}$ is the number of colors. Setting the net hypercharge density in the universe to zero, $Y=0$, gives

$$
c_{Y}=-\frac{8}{11} c_{B-L}
$$

The net $B-L$ number density in the visible sector (i.e. excluding the $B-L$ asymmetry carried by the $\chi$ fields in the dark sector) is then

$$
B-L \propto N_{f}\left(-2 \mu_{L}-\mu_{E}+2 \mu_{Q}+\mu_{U}+\mu_{D}\right)=\frac{79}{11} c_{B-L} .
$$

There are two types of interactions between the dark and visible sector: the asymmetric interactions that involve a single $\chi$ field, and the symmetric interactions of the form $\chi^{\dagger} \chi$ times the SM fields. The symmetric operators keep the dark and the visible sectors in thermal equilibrium. The asymmetric interactions are suppressed, and decouple at temperatures well above the $\chi$ mass. At lower temperatures the $\chi$ number is thus effectively conserved. The chemical potential $\mu_{\chi}$ is the same as it was before the decoupling. We thus have

$$
\mu_{\chi}^{i}=(B-L)_{\chi}^{i} c_{B-L}
$$

where $(B-L)_{\chi}^{i}$ is the $B-L$ charge of the $\chi^{i}$ field. Here we allow for several $\chi^{i}$ fields in the dark sector and also define the weighted $B-L$ charge of the dark sector fields as

$$
(B-L)_{\chi}^{\mathrm{sum}} \equiv \sum_{i} \hat{g}_{\chi}^{i}(B-L)_{\chi}^{i}
$$

The net $\chi$ number density normalized to entropy density we denote by $\Delta \chi$ and is

$$
\Delta \chi \propto \sum_{i} \hat{g}_{\chi}^{i} \mu_{\chi}^{i}=(B-L)_{\chi}^{\mathrm{sum}} c_{B-L}
$$

Since $B-L$ and $\chi$ are conserved quantum numbers below the decoupling temperature, each of the number densities scales as $R^{-3}$ as universe expands. The ratio

$$
\frac{\Delta \chi}{B-L}=\left.\frac{\Delta \chi}{B-L}\right|_{\text {decoup. }}=\frac{11}{79}(B-L)_{\chi}^{\text {sum }},
$$

thus stays fixed.

Even if at the decoupling there are more $\chi_{i}$ dark sector states, we assume that DM is composed only from one state, $\chi$. We therefore have for the ratio of baryon and dark matter energy densities

$$
\frac{\Omega_{B}}{\Omega_{\chi}}=\frac{m_{p}}{m_{\chi}} \frac{B}{B-L} \frac{B-L}{\Delta \chi} .
$$


The ratio of net $B$ and $B-L$ numbers $B /(B-L)=28 / 79=0.354$ just above the electroweak phase transition [89]. This remains essentially unchanged even if sphaleron and top mass effects are taken into account, in which case using results from $[88,91]$ one has $B /(B-L)=0.349$ for both scalar and fermionic DM. Using $(B-L) / \Delta \chi=$ $79 /\left(11(B-L)_{\chi}^{\text {sum }}\right)$ from (B.11) finally leads to

$$
m_{\chi}=2.509 m_{p} \frac{\Omega_{\chi}}{\Omega_{B}} \frac{1}{(B-L)_{\chi}^{\text {sum }}}=(12.5 \pm 0.8) \mathrm{GeV} \frac{1}{(B-L)_{\chi}^{\text {sum }}},
$$

where in the last equality we used $\Omega_{\chi}=0.265 \pm 0.011$ and $\Omega_{B}=0.0499 \pm 0.0022$ [53]. Note that the error is dominated by the experimental determination of DM and baryon densities. For instance, the difference between $B /(B-L)$ determination with and without sphaleron effects leads to a smaller shift in $m_{\chi}$ than the above quoted error.

We turn next to the case of additional fields in the visible sector. An example would be that SM gets completed to the MSSM. The relation between $Y, B-L$ and the constants $c_{Y, B-L}$ can be written in the matrix form

$$
\begin{aligned}
\left(\begin{array}{c}
Y \\
B-L
\end{array}\right) & =\frac{15}{4 \pi^{2} g_{*} T}\left(\begin{array}{cc}
\sum_{i} \hat{g}_{i} Y_{i}^{2} & \sum_{i} \hat{g}_{i} Y_{i}(B-L)_{i} \\
\sum_{i} \hat{g}_{i} Y_{i}(B-L)_{i} & \sum_{i} \hat{g}_{i}(B-L)_{i}^{2}
\end{array}\right) \cdot\left(\begin{array}{c}
c_{Y} \\
c_{B-L}
\end{array}\right) \\
& =\frac{15}{4 \pi^{2} g_{*} T}\left(\begin{array}{cc}
11+\left[Y^{2}\right]_{\mathrm{NP}} & 8+[Y(B-L)]_{\mathrm{NP}} \\
8+[Y(B-L)]_{\mathrm{NP}} & 13+\left[(B-L)^{2}\right]_{\mathrm{NP}}
\end{array}\right) \cdot\left(\begin{array}{c}
c_{Y} \\
c_{B-L}
\end{array}\right) .
\end{aligned}
$$

Here we defined

$$
\left[Y^{2}\right]_{\mathrm{NP}}=\sum_{i} \hat{g}_{i} Y_{i}^{2}, \quad[Y(B-L)]_{\mathrm{NP}}=\sum_{i} \hat{g}_{i} Y_{i}(B-L)_{i}, \quad\left[(B-L)^{2}\right]_{\mathrm{NP}}=\sum_{i} \hat{g}_{i}(B-L)_{i}^{2},
$$

where the sums run over the new states only. The solution for $B-L$ in terms of $c_{B-L}$ is obtained by solving the above matrix equation setting $Y=0$, from which

$$
B-L=\frac{15 c_{B-L}}{4 \pi^{2} g_{*} T}\left(13+\left[(B-L)^{2}\right]_{\mathrm{NP}}-\frac{\left(8+[Y(B-L)]_{\mathrm{NP}}\right)^{2}}{11+\left[Y^{2}\right]_{\mathrm{NP}}}\right) .
$$

The net $\chi$ charge is still given by eq. (B.11), while the ratio $\Omega_{B} / \Omega_{\chi}$ is given by (B.12) with $(B-L) / \Delta \chi$ fixed at the decoupling temperature and $B /(B-L)$ at the electroweak phase transition. We thus have

$$
m_{\chi}=m_{p} \frac{\Omega_{\chi}}{\Omega_{B}} \frac{B}{B-L}\left(13+\left[(B-L)^{2}\right]_{\mathrm{NP}}-\frac{\left(8+[Y(B-L)]_{\mathrm{NP}}\right)^{2}}{\left(11+\left[Y^{2}\right]_{\mathrm{NP}}\right)^{2}}\right) \frac{1}{(B-L)_{\chi}^{\text {sum }}},
$$

where $B /(B-L)=0.349$ and $(B-L)_{\chi}^{\text {sum }}$ given in (B.9).

\section{Calculation of the DM decay time}

Here we give further details of the DM lifetime calculation in the MFV and FN models for $B=2 \mathrm{DM}$, section 3, while also varying the DM mass. The results are shown in figure 3 . There are three different types of dimension 10 operators that can lead to DM decay, of 
schematic form $\chi\left(d^{c} d^{c}\right)\left(d^{c} d^{c}\right)\left(u^{c} u^{c}\right), \chi\left(d^{c} d^{c}\right)\left(d^{c} u^{c}\right)\left(q^{*} q^{*}\right)$, and $\chi\left(q^{*} q^{*}\right)\left(q^{*} q^{*}\right)\left(d^{c} d^{c}\right)$. For the same NP suppression scale $\Lambda$ the last type of operators gives the shortest lifetime. The dominant effective decay Lagrangian is thus, schematically,

$$
\mathcal{L}_{\text {dec }}^{(B=2)} \supset \frac{\mathcal{C}}{\Lambda^{6}} \chi\left(q^{*} q^{*}\right)\left(q^{*} q^{*}\right)\left(d^{c} d^{c}\right),
$$

where $\mathcal{C}$ is a flavor-dependent Wilson coefficient, the brackets enclose Lorentz contracted pairs, and summation over different flavor, color and weak isospin contractions is understood.

In section 3 we included the SM Yukawa insertions in the definition of the operators. To unify the notation we instead use in this appendix the convention that the Wilson coefficient $\mathcal{C}$ encodes all the flavor suppressions. The effective decay Lagrangian is thus, going to the mass basis, and displaying the flavor indices only,

$$
\mathcal{L}_{\text {dec. }}^{(B=2)} \supset \frac{\mathcal{C}^{\text {IJBCEF }}}{\Lambda^{6}} \chi u_{\mathrm{I}}^{*} u_{\mathrm{J}}^{*} d_{\mathrm{B}}^{*} d_{\mathrm{E}}^{*} d_{\mathrm{C}}^{c} d_{\mathrm{F}}^{c},
$$

where the flavor dependent Wilson coefficients are

$$
\begin{aligned}
& \mathcal{C}_{(\mathrm{MFV})}^{\mathrm{IJBCEF}} \simeq\left[V_{\mathrm{CKM}}\right]_{\mathrm{IA}}\left[Y_{d}^{\text {diag }}\right]_{\mathrm{B}}\left[V_{\mathrm{CKM}}\right]_{\mathrm{JD}} \varepsilon^{\mathrm{ABC}} \varepsilon^{\mathrm{DEF}}, \\
& \mathcal{C}_{(\mathrm{FN})}^{\mathrm{IJBCF}} \simeq \lambda^{\left|-H\left(q_{\mathrm{I}}\right)-H\left(q_{\mathrm{J}}\right)-H\left(q_{\mathrm{B}}\right)-H\left(q_{\mathrm{E}}\right)+H\left(d_{\mathrm{C}}^{c}\right)+H\left(d_{\mathrm{F}}^{c}\right)\right|},
\end{aligned}
$$

The partial decay width for $\chi \rightarrow q q q q d d$ transition is then, using NDA,

$$
\Gamma_{\chi} \simeq \frac{\mathcal{C}^{2}}{8 \pi} \frac{1}{\left(16 \pi^{2}\right)^{4}}\left(\frac{m_{\chi}}{\Lambda}\right)^{12} m_{\chi}
$$

The factor $1 /(8 \pi) \times 1 /\left(16 \pi^{2}\right)^{4}$ results from integrating over the 6 -body phase space.

For the MFV flavor breaking case there are several subtleties when calculating the decay width. For instance, the Levi-Civita tensor contractions lead to vanishing operators for some of the color and Lorentz contractions. Another subtlety is that the tree decay may be strongly CKM suppressed so that the leading decay amplitude is the 1-loop one, see figure 10. The decay width can thus be estimated as

$$
\Gamma\left(\chi \rightarrow u_{\mathrm{I}} u_{\mathrm{J}} d_{\mathrm{B}} d_{\mathrm{E}} d_{\mathrm{C}}^{c *} d_{\mathrm{F}}^{c *}\right) \simeq \max \left\{\begin{array}{l}
\frac{\left(\mathcal{C}_{(\mathrm{MFV})}^{\mathrm{IJBCEF}}\right)^{2}}{8 \pi}\left(\frac{1}{16 \pi^{2}}\right)^{4}\left(\frac{m_{\chi}}{\Lambda}\right)^{12} m_{\chi}, \\
\left(\frac{1}{16 \pi^{2}}\left[V_{\mathrm{CKM}}\right]_{\mathrm{IX}}\left[V_{\mathrm{CKM}}\right]_{\mathrm{YE}}\right)^{2} \frac{\left(\mathcal{C}_{(\mathrm{MFV})}^{\mathrm{YJBCXF}}\right)^{2}}{8 \pi}\left(\frac{1}{16 \pi^{2}}\right)^{4}\left(\frac{m_{\chi}}{\Lambda}\right)^{12} m_{\chi} .
\end{array}\right.
$$

where the first (second) line gives the NDA estimates for the tree level (1-loop) dominated decay width. The $W$ emitted from the left-handed quark lines coming from the effective decay vertex gives the additional CKM factors in the second line.

An example where the leading decay amplitude is generated at 1-loop is the $B=2$ ADM with $m_{\chi}=3.3 \mathrm{GeV}$, discussed in section 3. Decays into final states with one charm, bottom or top quark are kinematically forbidden. For instance, the lightest $B=2$ final states with one valence charm quark are $\Lambda_{c}^{+}+\Sigma^{-}$and $n+\Sigma_{c}^{0}$. The first has the valence quark content $\sim u d c+d d s$ and the rest mass $m_{\Lambda_{c}^{+}}+m_{\Sigma^{-}}=3.48 \mathrm{GeV}$, while the valence 

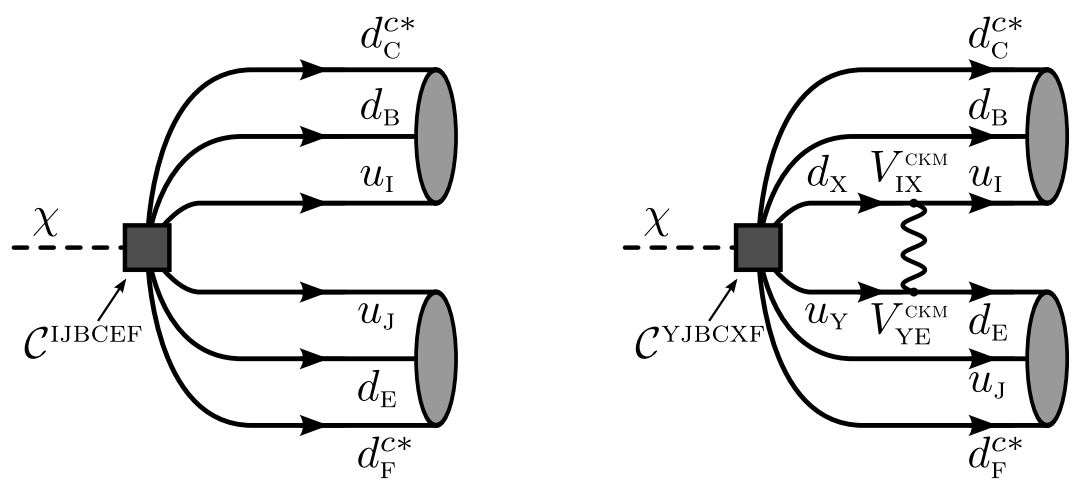

Figure 10. Example Feynman diagrams for the decay of $B=2 \mathrm{DM}$. The diagram on the left shows the tree level decay whereas the one on the right shows the loop-induced decay.

quark content of the second is $u d d+d d c$ and its rest mass $m_{n}+m_{\Sigma_{c}^{0}}=3.4 \mathrm{GeV}$. In contrast, the decays to $\Xi^{0}(\sim u d s)$ or $\Lambda^{0}(\sim u d s)$ baryons are allowed. Eq. (C.6) gives

$$
\begin{aligned}
& \Gamma_{\mathrm{TREE}}^{\mathrm{MFV}}\left(\chi \rightarrow \Lambda^{0} \Lambda^{0}\right) \simeq\left(y_{s} V_{u b}^{2}\right)^{2} \frac{1}{8 \pi}\left(\frac{1}{16 \pi^{2}}\right)^{4}\left(\frac{m_{\chi}}{\Lambda}\right)^{12} m_{\chi}, \\
& \Gamma_{\mathrm{LOOP}}^{\mathrm{MFV}}\left(\chi \rightarrow \Lambda^{0} \Lambda^{0}\right) \simeq\left(\frac{y_{b} V_{u b} V_{t s}}{16 \pi^{2}}\right)^{2} \frac{1}{8 \pi}\left(\frac{1}{16 \pi^{2}}\right)^{4}\left(\frac{m_{\chi}}{\Lambda}\right)^{12} m_{\chi},
\end{aligned}
$$

with the same estimate, within our precision, for the $\chi \rightarrow \Xi^{0}, \Xi^{0}$ or $\chi \rightarrow \Lambda^{0}, \Lambda^{0}$ decays. Note that in the 1-loop amplitude the partonic transition at the decay vertex, $\chi \rightarrow u d b+$ $t d s$, carries no CKM suppression. Furthermore, the $y_{s}$ Yukawa insertion in the tree level amplitude is replaced by $y_{b}$. The $b$ and $t$ quark lines then convert to $u$ and $s$ quark lines via $W$ exchange, as shown in figure 10. The smaller CKM and Yukawa suppressions compensate the loop factor so that the 1-loop amplitude dominates, with the NDA estimate $\Gamma_{\mathrm{LOOP}}^{\mathrm{MFV}} / \Gamma_{\mathrm{TREE}}^{\mathrm{MFV}} \sim \mathcal{O}(10)$

This procedure can be repeated for different DM masses, arriving at the dominant decay modes as a function of $m_{\chi}$. The results are listed in table 5, where we give the kinematical thresholds (1st column) for a number of decay channels (4th column), along with the corresponding partonic transitions (3rd column) and the decay vertex transitions (2nd column). The latter two differ for the loop processes, cf. figure 10. The total decay width for given $m_{\chi}$ is then the sum of partial decay widths, $\Gamma_{i}$, (5th column) for the decay channels that are kinematically allowed. For convenience we also give the decay times, $\tau_{i}$, (6th column) that correspond to individual partial decay widths. Note that in the calculation of the partial decay widths we neglect the phase space suppression, while the quoted $\Gamma_{i}$ in table 5 are obtained from the NDA estimates (C.6) with $m_{\chi}$ at the kinematical threshold, and setting $\Lambda=1 \mathrm{TeV}$.

In the case of FN flavor breaking the leading tree level and loop induced decay widths 


\begin{tabular}{|c|c|c|c|c|c|c|}
\hline Thr. [GeV] & Decay vertex & Partonic & Final State & $\overline{\Gamma_{i}[\mathrm{GeV}]}$ & $\tau_{i}[\mathrm{~s}]$ & Process \\
\hline 2.06 & $\chi \rightarrow c u d b s d$ & $\chi \rightarrow u d d u d s$ & $n+\Lambda^{0}$ & $1.34 \times 10^{-60}$ & $4.91 \times 10^{35}$ & Loop \\
\hline 2.23 & $\chi \rightarrow$ uusdsd & $\chi \rightarrow u d s u d s$ & $\Lambda^{0}+\Lambda^{0}$ & $3.74 \times 10^{-55}$ & $1.76 \times 10^{30}$ & Tree \\
\hline 2.43 & $\chi \rightarrow$ cusbsd & $\chi \rightarrow$ udsuss & $\Lambda^{0}+\Xi^{0}$ & $4.25 \times 10^{-57}$ & $1.55 \times 10^{32}$ & Loop \\
\hline 3.48 & $\chi \rightarrow c c d s s d$ & $\chi \rightarrow u d c d d s$ & $\Lambda_{c}^{+}+\Sigma^{-}$ & $1.23 \times 10^{-55}$ & $5.33 \times 10^{30}$ & Loop \\
\hline 3.61 & $\chi \rightarrow u c s d s d$ & $\chi \rightarrow u d c d s s$ & $\Lambda_{c}^{+}+\Xi^{-}$ & $1.87 \times 10^{-50}$ & $3.52 \times 10^{25}$ & Tree \\
\hline 3.81 & $\chi \rightarrow c c s d s d$ & $\chi \rightarrow u d s s s c$ & $\Lambda^{0}+\Omega_{c}^{0}$ & $1.42 \times 10^{-52}$ & $4.62 \times 10^{27}$ & Loop \\
\hline 3.79 & $\chi \rightarrow c c s d s d$ & $\chi \rightarrow u s c d s s$ & $\Xi_{c}^{+}+\Xi^{-}$ & $1.33 \times 10^{-52}$ & $4.96 \times 10^{27}$ & Loop \\
\hline 4.63 & $\chi \rightarrow c c d b s d$ & $\chi \rightarrow d c c d d s$ & $\Xi_{c c}^{+}+\Sigma^{-}$ & $1.01 \times 10^{-52}$ & $6.51 \times 10^{27}$ & Loop \\
\hline 4.93 & $\chi \rightarrow c c s d s d$ & $\chi \rightarrow d d c d s c$ & $\Sigma_{c}^{0}+\Xi_{c}^{0}$ & $1.04 \times 10^{-46}$ & $6.33 \times 10^{21}$ & Tree \\
\hline 5.17 & $\chi \rightarrow c c s b s d$ & $\chi \rightarrow d s c s s c$ & $\Xi_{c}^{0}+\Omega_{c}^{0}$ & $4.14 \times 10^{-52}$ & $1.59 \times 10^{27}$ & Loop \\
\hline 6.56 & $\chi \rightarrow c u d s b d$ & $\chi \rightarrow u d d u d b$ & $n+\Lambda_{b}^{0}$ & $7.25 \times 10^{-54}$ & $9.08 \times 10^{28}$ & Loop \\
\hline 6.73 & $\chi \rightarrow$ uudbsd & $\chi \rightarrow u d d u s b$ & $n+\Xi_{b}^{0}$ & $1.87 \times 10^{-45}$ & $3.53 \times 10^{20}$ & Tree \\
\hline 6.94 & $\chi \rightarrow$ uusbsd & $\chi \rightarrow u s s u d b$ & $\Xi^{0}+\Lambda_{b}^{0}$ & $5.18 \times 10^{-44}$ & $1.27 \times 10^{19}$ & Tree \\
\hline 7.10 & $\chi \rightarrow c u s d b s$ & $\chi \rightarrow$ uss usb & $\Xi^{0}+\Xi_{b}^{0}$ & $3.49 \times 10^{-52}$ & $1.89 \times 10^{27}$ & Loop \\
\hline 8.07 & $\chi \rightarrow u c b d b d$ & $\chi \rightarrow u d b d d c$ & $\Lambda_{b}^{0}+\Sigma_{c}^{0}$ & $2.16 \times 10^{-51}$ & $3.05 \times 10^{26}$ & Loop \\
\hline 8.09 & $\chi \rightarrow u c b d s d$ & $\chi \rightarrow u d b d s c$ & $\Lambda_{b}^{0}+\Xi_{c}^{0}$ & $2.00 \times 10^{-42}$ & $3.30 \times 10^{17}$ & Tree \\
\hline 8.31 & $\chi \rightarrow u c b s s d$ & $\chi \rightarrow u d b s s c$ & $\Lambda_{b}^{0}+\Omega_{c}^{0}$ & $5.33 \times 10^{-41}$ & $1.24 \times 10^{16}$ & Tree \\
\hline 8.48 & $\chi \rightarrow u c b s b s$ & $\chi \rightarrow u s b s s c$ & $\Xi_{b}^{0}+\Omega_{c}^{0}$ & $7.66 \times 10^{-50}$ & $8.59 \times 10^{24}$ & Loop \\
\hline 11.24 & $\chi \rightarrow u u b d b d$ & $\chi \rightarrow u d b u d b$ & $\Lambda_{b}^{0}+\Lambda_{b}^{0}$ & $2.24 \times 10^{-42}$ & $2.93 \times 10^{17}$ & Tree \\
\hline 11.41 & $\chi \rightarrow u u b b s d$ & $\chi \rightarrow u d b u s b$ & $\Lambda_{b}^{0}+\Xi_{b}^{0}$ & $9.86 \times 10^{-38}$ & $6.68 \times 10^{12}$ & Tree \\
\hline 11.58 & $\chi \rightarrow u u b s b s$ & $\chi \rightarrow$ usbusb & $\Xi_{b}^{0}+\Xi_{b}^{0}$ & $2.94 \times 10^{-42}$ & $2.24 \times 10^{17}$ & Tree \\
\hline
\end{tabular}

Table 5. Partial decay widths, $\Gamma_{i}$, and related decay times, $\tau_{i}=1 / \Gamma_{i}$, for representative decay channels above kinematical thresholds (1st column) assuming the MFV flavor breaking ansatz. The EFT scale is set to $\Lambda=1 \mathrm{TeV}$. The last column denotes whether the dominant amplitude is tree level or 1-loop, while the 2nd and the 3rd columns give the decay vertex transition and the partonic transition after the potential $W$ exchange, respectively.

for $B=2, m_{\chi}=3.3 \mathrm{GeV}$ DM have the NDA estimates of

$$
\begin{aligned}
& \Gamma_{\mathrm{TREE}}^{\mathrm{FN}}\left(\chi \rightarrow \Lambda^{0} \Lambda^{0}\right) \simeq\left(\lambda^{2}\right)^{2} \frac{1}{8 \pi}\left(\frac{1}{16 \pi^{2}}\right)^{4}\left(\frac{m_{\chi}}{\Lambda}\right)^{12} m_{\chi} \\
& \Gamma_{\mathrm{LOOP}}^{\mathrm{FN}}\left(\chi \rightarrow \Lambda^{0} \Lambda^{0}\right) \simeq\left(\frac{\lambda^{4}}{16 \pi^{2}}\right)^{2} \frac{1}{8 \pi}\left(\frac{1}{16 \pi^{2}}\right)^{4}\left(\frac{m_{\chi}}{\Lambda}\right)^{12} m_{\chi} .
\end{aligned}
$$

In this case the tree level decay dominates over the loop induced decay by four orders of magnitude. The dominance of the tree level decay amplitude over the 1-loop decay amplitude holds also, if the DM mass is varied. This can be traced to the following difference between the MFV and FN ansätze. In the MFV case the Levi-Civita tensors enforce that two quark flavors in the effective decay vertex need to be from the third generation. This can be changed either by using the $V_{\mathrm{CKM}}$ misalignment or through a loop transition. In FN flavor structure ansatz, on the other hand, the flavor indices need not be antisymmetric. 


\section{Loop functions in neutral meson mixing}

Here we list the analytical form of the loop functions $F(x), F^{F}\left(x_{1}, x_{2}\right), G\left(x_{1}, x_{2}\right), G^{F}\left(x_{1}, x_{2}, x 3\right)$ and $H(x), H^{F}\left(x_{1}, x_{2}\right)$ that appear in the 1-loop expressions for the Wilson coefficients in the neutral meson mixing, section 6.1. The mediator loop functions with mass degenerate quarks in the loop are given by

$$
F(x)=x H(x), \quad H(x)=\frac{1}{(1-x)^{3}}\left[1-x^{2}+2 x \log (x)\right],
$$

while for two different quarks running in the loop they are

$$
\begin{aligned}
& F^{F}\left(x_{i}, x_{j}\right)=\frac{x_{i} x_{j}}{\left(1-x_{i}\right)\left(1-x_{j}\right)}+\left[\frac{x_{i} x_{j} \log \left(x_{i}\right)}{\left(1-x_{i}\right)^{2}\left(x_{i}-x_{j}\right)}+x_{i} \leftrightarrow x_{j}\right], \\
& H^{F}\left(x_{i}, x_{j}\right)=\frac{1}{\left(1-x_{i}\right)\left(1-x_{j}\right)}+\left[\frac{x_{i}^{2} \log \left(x_{i}\right)}{\left(1-x_{i}\right)^{2}\left(x_{i}-x_{j}\right)}+x_{i} \leftrightarrow x_{j}\right] .
\end{aligned}
$$

The loop functions for the mediator- $W$ loops are

$$
\begin{gathered}
G\left(x_{w}, x\right)=\frac{x}{x_{w}}\left[\frac{x_{w}+x}{(x-1)\left(x-x_{w}\right)}-\frac{x\left(2 x x_{w}-x_{w}^{2}-2 x_{w}+x^{2}\right) \log (x)}{(x-1)^{2}\left(x-x_{w}\right)^{2}}\right. \\
\left.+\frac{2 x x_{w} \log \left(x_{w}\right)}{\left(x-x_{w}\right)^{2}\left(x_{w}-1\right)}\right], \\
G^{F}\left(x_{w}, x_{i}, x_{j}\right)=\frac{x_{i} x_{j}}{x_{w}}\left[\left\{\frac{\left(x_{i}+x_{w}\right) \log \left(x_{i}\right)}{\left(1-x_{i}\right)\left(x_{i}-x_{j}\right)\left(x_{w}-x_{i}\right)}+x_{i} \leftrightarrow x_{j}\right\}\right. \\
\left.-\frac{2 x_{w} \log \left(x_{w}\right)}{\left(1-x_{w}\right)\left(x_{w}-x_{i}\right)\left(x_{w}-x_{j}\right)}\right] .
\end{gathered}
$$

Open Access. This article is distributed under the terms of the Creative Commons Attribution License (CC-BY 4.0), which permits any use, distribution and reproduction in any medium, provided the original author(s) and source are credited.

\section{References}

[1] T. Hambye, On the stability of particle dark matter, PoS(IDM2010) 098 [arXiv:1012.4587] [INSPIRE].

[2] L. Ackerman, M.R. Buckley, S.M. Carroll and M. Kamionkowski, Dark Matter and Dark Radiation, Phys. Rev. D 79 (2009) 023519 [arXiv:0810.5126] [INSPIRE].

[3] J.L. Feng, H. Tu and H.-B. Yu, Thermal Relics in Hidden Sectors, JCAP 10 (2008) 043 [arXiv: 0808.2318] [INSPIRE].

[4] J.L. Feng, M. Kaplinghat, H. Tu and H.-B. Yu, Hidden Charged Dark Matter, JCAP 07 (2009) 004 [arXiv: 0905.3039] [INSPIRE].

[5] G.R. Farrar and P. Fayet, Phenomenology of the Production, Decay and Detection of New Hadronic States Associated with Supersymmetry, Phys. Lett. B 76 (1978) 575 [INSPIRE]. 
[6] S. Dimopoulos, S. Raby and F. Wilczek, Proton Decay in Supersymmetric Models, Phys. Lett. B 112 (1982) 133 [INSPIRE].

[7] G.R. Farrar and S. Weinberg, Supersymmetry at Ordinary Energies. 2. R Invariance, Goldstone Bosons and Gauge Fermion Masses, Phys. Rev. D 27 (1983) 2732 [InSPIRE].

[8] M. Kadastik, K. Kannike and M. Raidal, Matter parity as the origin of scalar Dark Matter, Phys. Rev. D 81 (2010) 015002 [arXiv: 0903.2475] [inSPIRE].

[9] M. Kadastik, K. Kannike and M. Raidal, Dark Matter as the signal of Grand Unification, Phys. Rev. D 80 (2009) 085020 [Erratum ibid. D 81 (2010) 029903] [arXiv:0907.1894] [INSPIRE].

[10] M. Frigerio and T. Hambye, Dark matter stability and unification without supersymmetry, Phys. Rev. D 81 (2010) 075002 [arXiv:0912.1545] [INSPIRE].

[11] M. Cirelli and A. Strumia, Minimal Dark Matter: Model and results, New J. Phys. 11 (2009) 105005 [arXiv: 0903.3381] [INSPIRE].

[12] M. Cirelli, N. Fornengo and A. Strumia, Minimal dark matter, Nucl. Phys. B 753 (2006) 178 [hep-ph/0512090] [INSPIRE].

[13] T. Hambye, Hidden vector dark matter, JHEP 01 (2009) 028 [arXiv:0811.0172] [INSPIRE].

[14] Y. Bai and R.J. Hill, Weakly Interacting Stable Pions, Phys. Rev. D 82 (2010) 111701 [arXiv: 1005.0008] [INSPIRE].

[15] D.E. Kaplan, M.A. Luty and K.M. Zurek, Asymmetric Dark Matter, Phys. Rev. D 79 (2009) 115016 [arXiv: 0901.4117] [INSPIRE].

[16] K.M. Zurek, Asymmetric Dark Matter: Theories, Signatures and Constraints, Phys. Rept. 537 (2014) 91 [arXiv: 1308.0338] [INSPIRE].

[17] K. Petraki and R.R. Volkas, Review of asymmetric dark matter, Int. J. Mod. Phys. A 28 (2013) 1330028 [arXiv:1305.4939] [INSPIRE].

[18] B. Batell, J. Pradler and M. Spannowsky, Dark Matter from Minimal Flavor Violation, JHEP 08 (2011) 038 [arXiv:1105.1781] [INSPIRE].

[19] J. Kile and A. Soni, Flavored Dark Matter in Direct Detection Experiments and at LHC, Phys. Rev. D 84 (2011) 035016 [arXiv:1104.5239] [inSPIRE].

[20] P. Agrawal, S. Blanchet, Z. Chacko and C. Kilic, Flavored Dark Matter and Its Implications for Direct Detection and Colliders, Phys. Rev. D 86 (2012) 055002 [arXiv:1109.3516] [INSPIRE].

[21] H. Davoudiasl, D.E. Morrissey, K. Sigurdson and S. Tulin, Baryon Destruction by Asymmetric Dark Matter, Phys. Rev. D 84 (2011) 096008 [arXiv:1106.4320] [InSPIRE].

[22] L. Lopez-Honorez and L. Merlo, Dark matter within the minimal flavour violation ansatz, Phys. Lett. B 722 (2013) 135 [arXiv:1303.1087] [InSPIRE].

[23] P. Agrawal, M. Blanke and K. Gemmler, Flavored dark matter beyond Minimal Flavor Violation, JHEP 1410 (2014) 72 [arXiv: 1405.6709] [INSPIRE].

[24] B. Batell, T. Lin and L.-T. Wang, Flavored Dark Matter and R-Parity Violation, JHEP 01 (2014) 075 [arXiv:1309.4462] [INSPIRE].

[25] P. Agrawal, B. Batell, D. Hooper and T. Lin, Flavored Dark Matter and the Galactic Center Gamma-Ray Excess, Phys. Rev. D 90 (2014) 063512 [arXiv:1404.1373] [InSPIRE]. 
[26] U. Haisch, A. Hibbs and E. Re, Determining the structure of dark-matter couplings at the LHC, Phys. Rev. D 89 (2014) 034009 [arXiv:1311.7131] [InSPIRE].

[27] J.F. Kamenik and J. Zupan, Discovering Dark Matter Through Flavor Violation at the LHC, Phys. Rev. D 84 (2011) 111502 [arXiv:1107.0623] [InSPIRE].

[28] A. Merle and V. Niro, Deriving Models for keV sterile Neutrino Dark Matter with the Froggatt-Nielsen mechanism, JCAP 07 (2011) 023 [arXiv: 1105.5136] [INSPIRE].

[29] Y. Kajiyama, K. Kannike and M. Raidal, Symmetryless Dark Matter, Phys. Rev. D 85 (2012) 033008 [arXiv:1111.1270] [INSPIRE].

[30] M. Lattanzi, R.A. Lineros and M. Taoso, Connecting neutrino physics with dark matter, arXiv: 1406.0004 [INSPIRE].

[31] M. Hirsch, S. Morisi, E. Peinado and J.W.F. Valle, Discrete dark matter, Phys. Rev. D 82 (2010) 116003 [arXiv:1007.0871] [INSPIRE].

[32] M.S. Boucenna, S. Morisi, E. Peinado, Y. Shimizu and J.W.F. Valle, Predictive discrete dark matter model and neutrino oscillations, Phys. Rev. D 86 (2012) 073008 [arXiv:1204.4733] [INSPIRE].

[33] H. An, S.-L. Chen, R.N. Mohapatra and Y. Zhang, Leptogenesis as a Common Origin for Matter and Dark Matter, JHEP 03 (2010) 124 [arXiv:0911.4463] [INSPIRE].

[34] K. Agashe and G. Servant, Warped unification, proton stability and dark matter, Phys. Rev. Lett. 93 (2004) 231805 [hep-ph/0403143] [INSPIRE].

[35] Y. Zhao and K.M. Zurek, Indirect Detection Signatures for the Origin of Asymmetric Dark Matter, JHEP 07 (2014) 017 [arXiv:1401.7664] [INSPIRE].

[36] L. Feng and Z. Kang, Decaying Asymmetric Dark Matter Relaxes the AMS-Fermi Tension, JCAP 10 (2013) 008 [arXiv: 1304.7492] [INSPIRE].

[37] I. Masina, P. Panci and F. Sannino, Gamma Ray Constraints on Flavor Violating Asymmetric Dark Matter, JCAP 12 (2012) 002 [arXiv:1205.5918] [INSPIRE].

[38] I. Masina and F. Sannino, Charge Asymmetric Cosmic Rays as a probe of Flavor Violating Asymmetric Dark Matter, JCAP 09 (2011) 021 [arXiv:1106.3353] [INSPIRE].

[39] S.M. Barr, R.S. Chivukula and E. Farhi, Electroweak Fermion Number Violation and the Production of Stable Particles in the Early Universe, Phys. Lett. B 241 (1990) 387 [INSPIRE].

[40] D.B. Kaplan, A Single explanation for both the baryon and dark matter densities, Phys. Rev. Lett. 68 (1992) 741 [INSPIRE].

[41] S.M. Barr, Baryogenesis, sphalerons and the cogeneration of dark matter, Phys. Rev. D 44 (1991) 3062 [INSPIRE].

[42] S. Dodelson, B.R. Greene and L.M. Widrow, Baryogenesis, dark matter and the width of the Z, Nucl. Phys. B 372 (1992) 467 [inSPIRE].

[43] V.A. Kuzmin, A Simultaneous solution to baryogenesis and dark matter problems, Phys. Part. Nucl. 29 (1998) 257 [hep-ph/9701269] [INSPIRE].

[44] M. Fujii and T. Yanagida, A Solution to the coincidence puzzle of Omega(B) and Omega (DM), Phys. Lett. B 542 (2002) 80 [hep-ph/0206066] [INSPIRE].

[45] D. Hooper, J. March-Russell and S.M. West, Asymmetric sneutrino dark matter and the Omega(b)/Omega(DM) puzzle, Phys. Lett. B 605 (2005) 228 [hep-ph/0410114] [InSPIRE]. 
[46] R. Kitano and I. Low, Dark matter from baryon asymmetry, Phys. Rev. D 71 (2005) 023510 [hep-ph/0411133] [INSPIRE].

[47] S.B. Gudnason, C. Kouvaris and F. Sannino, Towards working technicolor: Effective theories and dark matter, Phys. Rev. D 73 (2006) 115003 [hep-ph/0603014] [InSPIRE].

[48] R. Kitano, H. Murayama and M. Ratz, Unified origin of baryons and dark matter, Phys. Lett. B 669 (2008) 145 [arXiv: 0807.4313] [INSPIRE].

[49] L.J. Hall, J. March-Russell and S.M. West, A Unified Theory of Matter Genesis: Asymmetric Freeze-In, arXiv:1010.0245 [INSPIRE].

[50] J. March-Russell and M. McCullough, Asymmetric Dark Matter via Spontaneous Co-Genesis, JCAP 03 (2012) 019 [arXiv:1106.4319] [INSPIRE].

[51] M.L. Graesser, I.M. Shoemaker and L. Vecchi, Asymmetric WIMP dark matter, JHEP 10 (2011) 110 [arXiv:1103.2771] [INSPIRE].

[52] S. Nussinov, Technocosmology: Could A Technibaryon Excess Provide A 'natural' Missing Mass Candidate?, Phys. Lett. B 165 (1985) 55 [INSPIRE].

[53] Particle Data Group collaboration, K. Olive et al., Review of Particle Physics, Chin. Phys. C38 (2014) 090001.

[54] Planck collaboration, P.A.R. Ade et al., Planck 2013 results. XVI. Cosmological parameters, Astron. Astrophys. 571 (2014) A16 [arXiv:1303.5076] [INSPIRE].

[55] A. Falkowski, J.T. Ruderman and T. Volansky, Asymmetric Dark Matter from Leptogenesis, JHEP 05 (2011) 106 [arXiv: 1101.4936] [INSPIRE].

[56] Y. Cui, L. Randall and B. Shuve, Emergent Dark Matter, Baryon and Lepton Numbers, JHEP 08 (2011) 073 [arXiv:1106.4834] [INSPIRE].

[57] T. Lin, H.-B. Yu and K.M. Zurek, On Symmetric and Asymmetric Light Dark Matter, Phys. Rev. D 85 (2012) 063503 [arXiv:1111.0293] [InSPIRE].

[58] T. Cohen, D.J. Phalen, A. Pierce and K.M. Zurek, Asymmetric Dark Matter from a GeV Hidden Sector, Phys. Rev. D 82 (2010) 056001 [arXiv:1005.1655] [InSPIRE].

[59] M. Blennow, E. Fernandez-Martinez, O. Mena, J. Redondo and P. Serra, Asymmetric Dark Matter and Dark Radiation, JCAP 07 (2012) 022 [arXiv:1203.5803] [INSPIRE].

[60] J. March-Russell, J. Unwin and S.M. West, Closing in on Asymmetric Dark Matter I: Model independent limits for interactions with quarks, JHEP 08 (2012) 029 [arXiv:1203.4854] [INSPIRE].

[61] R.S. Chivukula and H. Georgi, Composite Technicolor Standard Model, Phys. Lett. B 188 (1987) 99 [INSPIRE].

[62] G. D'Ambrosio, G.F. Giudice, G. Isidori and A. Strumia, Minimal flavor violation: An Effective field theory approach, Nucl. Phys. B 645 (2002) 155 [hep-ph/0207036] [INSPIRE].

[63] L.J. Hall and L. Randall, Weak scale effective supersymmetry, Phys. Rev. Lett. 65 (1990) 2939 [INSPIRE].

[64] A.J. Buras, Minimal flavor violation, Acta Phys. Polon. B 34 (2003) 5615 [hep-ph/0310208] [INSPIRE].

[65] A.J. Buras, P. Gambino, M. Gorbahn, S. Jager and L. Silvestrini, Universal unitarity triangle and physics beyond the standard model, Phys. Lett. B 500 (2001) 161 [hep-ph/0007085] [INSPIRE]. 
[66] C.D. Froggatt and H.B. Nielsen, Hierarchy of Quark Masses, Cabibbo Angles and CP-violation, Nucl. Phys. B 147 (1979) 277 [inSPIRE].

[67] M. Leurer, Y. Nir and N. Seiberg, Mass matrix models: The Sequel, Nucl. Phys. B 420 (1994) 468 [hep-ph/9310320] [INSPIRE].

[68] LAT collaboration, M. Ackermann et al., Constraints on the Galactic Halo Dark Matter from Fermi-LAT Diffuse Measurements, Astrophys. J. 761 (2012) 91 [arXiv:1205.6474] [INSPIRE].

[69] AMS collaboration, M. Aguilar et al., First Result from the Alpha Magnetic Spectrometer on the International Space Station: Precision Measurement of the Positron Fraction in Primary Cosmic Rays of 0.5-350 GeV, Phys. Rev. Lett. 110 (2013) 141102.

[70] A. Ibarra, A.S. Lamperstorfer and J. Silk, Dark matter annihilations and decays after the AMS-02 positron measurements, Phys. Rev. D 89 (2014) 063539 [arXiv:1309.2570] [INSPIRE].

[71] Super-Kamiokande collaboration, S. Desai et al., Search for dark matter WIMPs using upward through-going muons in Super-Kamiokande, Phys. Rev. D 70 (2004) 083523 [Erratum ibid. D 70 (2004) 109901] [hep-ex/0404025] [INSPIRE].

[72] L. Covi, M. Grefe, A. Ibarra and D. Tran, Neutrino Signals from Dark Matter Decay, JCAP 04 (2010) 017 [arXiv: 0912.3521] [INSPIRE].

[73] Fermi-LAT collaboration, Fermi-LAT Observations of the Diffuse Gamma-Ray Emission: Implications for Cosmic Rays and the Interstellar Medium, Astrophys. J. 750 (2012) 3 [arXiv:1202.4039] [INSPIRE].

[74] LAT collaboration, M. Ackermann et al., Fermi LAT Search for Dark Matter in Gamma-ray Lines and the Inclusive Photon Spectrum, Phys. Rev. D 86 (2012) 022002 [arXiv:1205.2739] [INSPIRE].

[75] Fermi-LAT collaboration, A.A. Abdo et al., The Spectrum of the Isotropic Diffuse Gamma-Ray Emission Derived From First-Year Fermi Large Area Telescope Data, Phys. Rev. Lett. 104 (2010) 101101 [arXiv:1002.3603] [INSPIRE].

[76] T. Han, I. Lewis and T. McElmurry, QCD Corrections to Scalar Diquark Production at Hadron Colliders, JHEP 01 (2010) 123 [arXiv:0909.2666] [INSPIRE].

[77] B. Bhattacherjee, S. Matsumoto, S. Mukhopadhyay and M.M. Nojiri, Phenomenology of light fermionic asymmetric dark matter, JHEP 10 (2013) 032 [arXiv:1306.5878] [INSPIRE].

[78] G.F. Giudice, B. Gripaios and R. Sundrum, Flavourful Production at Hadron Colliders, JHEP 08 (2011) 055 [arXiv: 1105.3161] [INSPIRE].

[79] B. Grinstein, A.L. Kagan, J. Zupan and M. Trott, Flavor Symmetric Sectors and Collider Physics, JHEP 10 (2011) 072 [arXiv:1108.4027] [inSPIRE].

[80] UTFIT collaboration, M. Bona et al., Model-independent constraints on $\Delta F=2$ operators and the scale of new physics, JHEP 03 (2008) 049 [arXiv:0707.0636] [INSPIRE].

[81] J. Charles et al., Future sensitivity to new physics in $B_{d}, B_{s}$ and $K$ mixings, Phys. Rev. D 89 (2014) 033016 [arXiv: 1309.2293] [INSPIRE].

[82] CMS collaboration, S. Chatrchyan et al., Search for pair-produced dijet resonances in four-jet final states in pp collisions at $\sqrt{s}=7$ TeV, Phys. Rev. Lett. 110 (2013) 141802 [arXiv: 1302.0531] [INSPIRE]. 
[83] CMS collaboration, Search for direct production of bottom squark pairs, CMS-PAS-SUS-13-018 (2014).

[84] ATLAS and CMS Collaborations, Combination of single top-quark cross-sections measurements in the t-channel at $\sqrt{s}=8 \mathrm{TeV}$ with the ATLAS and CMS experiments, CMS-PAS-TOP-12-002 (2013).

[85] J. Alwall et al., The automated computation of tree-level and next-to-leading order differential cross sections and their matching to parton shower simulations, JHEP 07 (2014) 079 [arXiv: 1405.0301] [INSPIRE].

[86] A. Alloul, N.D. Christensen, C. Degrande, C. Duhr and B. Fuks, FeynRules 2.0 - A complete toolbox for tree-level phenomenology, Comput. Phys. Commun. 185 (2014) 2250 [arXiv: 1310.1921] [INSPIRE].

[87] H.K. Dreiner, H.E. Haber and S.P. Martin, Two-component spinor techniques and Feynman rules for quantum field theory and supersymmetry, Phys. Rept. 494 (2010) 1 [arXiv: 0812.1594] [INSPIRE].

[88] M. D'Onofrio, K. Rummukainen and A. Tranberg, The sphaleron rate at the electroweak crossover with $125 \mathrm{GeV}$ Higgs mass, PoS (LATTICE 2012) 055 [arXiv: 1212.3206] [INSPIRE].

[89] J.A. Harvey and M.S. Turner, Cosmological baryon and lepton number in the presence of electroweak fermion number violation, Phys. Rev. D 42 (1990) 3344 [INSPIRE].

[90] B. Feldstein and A.L. Fitzpatrick, Discovering Asymmetric Dark Matter with Anti-Neutrinos, JCAP 09 (2010) 005 [arXiv: 1003.5662] [INSPIRE].

[91] Y. Burnier, M. Laine and M. Shaposhnikov, Baryon and lepton number violation rates across the electroweak crossover, JCAP 02 (2006) 007 [hep-ph/0511246] [INSPIRE]. 